\title{
TITLE:
}

\section{Space representation in the prefrontal cortex.}

\author{
$\operatorname{AUTHOR}(S)$ :
}

Funahashi, Shintaro

\section{CITATION:}

Funahashi, Shintaro. Space representation in the prefrontal cortex..

Progress in neurobiology 2013, 103: 131-155

\section{ISSUE DATE:}

2013-04

URL:

http://hdl.handle.net/2433/173914

\section{RIGHT:}

(C) 2012 Elsevier Ltd.; This is not the published version. Please cite only the published version.; この論文は出版社版でありません。引用の際に は出版社版をご確認ご利用ください。 


\section{Space representation in the prefrontal cortex}

Shintaro Funahashi

Kokoro Research Center, Kyoto University

and

Department of Cognitive and Behavioral Sciences,

Graduate School of Human and Environmental Studies,

Kyoto University

Sakyo-ku, Kyoto 606-8501, Japan.

Phone \& Fax: (+81) 75-753-9674

E-mail: funahashi.shintaro.2z@kyoto-u.ac.jp

Correspondence:

Shintaro Funahashi, Ph.D.

Kokoro Research Center

Kyoto University

46 Yoshida-Shimoadachi, Sakyo-ku, Kyoto 606-8501, Japan.

Phone \& Fax: (+81) 75-753-9674

E-mail: funahashi.shintaro.2z@kyoto-u.ac.jp 


\section{Abstract}

The representation of space and its function in the prefrontal cortex have been examined using a variety of behavioral tasks. Among them, since the delayed-response task requires the temporary maintenance of spatial information, this task has been used to examine the mechanisms of spatial representation. In addition, the concept of working memory to explain prefrontal functions has helped us to understand the nature and functions of space representation in the prefrontal cortex. The detailed analysis of delay-period activity observed in spatial working memory tasks has provided important information for understanding space representation in the prefrontal cortex. Directional delay-period activity has been shown to be a neural correlate of the mechanism for temporarily maintaining information and represent spatial information for the visual cue and the saccade. In addition, many task-related prefrontal neurons exhibit spatially selective activities. These neurons are also important components of spatial information processing. In fact, information flow from sensory-related neurons to motor-related neurons has been demonstrated, along with a change in spatial representation as the trial progresses. The dynamic functional interactions among neurons exhibiting different task-related activities and representing different aspects of information could play an essential role in information processing. In addition, information provided from other cortical or subcortical areas might also be necessary for the representation of space in the prefrontal cortex. To better understand the representation of space and its function in the prefrontal cortex, we need to understand the nature of functional interactions between the prefrontal cortex and other cortical and subcortical areas. 


\section{Introduction}

The prefrontal cortex, and especially the dorsolateral prefrontal cortex, has been shown to participate in spatial information processing. This has been demonstrated by lesion studies in which monkeys with bilateral lesions in the dorsolateral prefrontal cortex exhibited severe impairments in the performance of the delayed-response task and the spatial delayed alternation task, but not in the visual discrimination task, the delayed matching-to-sample task, or the delayed object alternation task (e.g., Fuster 2008; Goldman-Rakic 1987; Rosenkilde 1979). In the former tasks, subjects are required to maintain spatial information regarding the visual cue to make a correct behavioral response, while in the latter tasks, subjects are required to maintain non-spatial information regarding the visual cue (e.g., the object itself or the color or shape of the object). Therefore, the dorsolateral prefrontal cortex has been thought to play an important role in maintaining spatial information and using this information to guide a correct response. This prefrontal function has been referred to as spatial working memory by Goldman-Rakic $(1987,1996 a)$.

Prefrontal participation in spatial working memory processes has been examined extensively. In addition to lesions, temporary dysfunction caused by a local injection of chemical (e.g., muscimol) into the prefrontal cortex has also been shown to impair visuospatial working memory in monkeys (Sawaguchi and Iba 2001). Further, functional brain imaging studies using human subjects have shown activation of the lateral prefrontal cortex while subjects performed spatial working memory tasks (D’Espositio et al. 1998; D’Esposito et al. 2000; Jonides et al. 1993; McCarthy et al. 1994). Evidence that the prefrontal cortex participates in the spatial working memory process has been obtained not only in monkey experiments, but also in rat experiments (Horst and Laubach 2009).

Neurophysiological studies using monkeys have shown that many prefrontal 
neurons exhibit tonic sustained excitatory activity during the delay period of spatial working memory tasks, and this activity has been called delay-period activity (e.g., Funahashi et al. 1989). Based on the characteristics of delay-period activity, this activity has been considered to be a neural correlate of a mechanism for temporarily maintaining information (Funahashi 2001; Funahashi and Kubota 1994; Fuster 2008; Goldman-Rakic 1987; Miller 2000; Miller and Cohen 2001; Petrides 1994). Persistent activity during the delay period has also been observed during spatial working memory performance in human functional brain imaging studies (Curtis and D'Esposito 2003). Thus, the prefrontal cortex, especially the dorsolateral prefrontal cortex, plays an important role in spatial working memory processes. Persistent delay-period activity is an important neural component for understanding spatial representation by prefrontal neurons. Therefore, a detailed examination of the characteristics of delay-period activity should provide important information regarding how prefrontal neurons represent and process spatial information.

However, delay-period activity can be observed not only during spatial working memory tasks, but also during non-spatial working memory tasks. In addition, delay-period activity participates not only in working memory processes, but also in the processing of information for other cognitive functions. These include the prospective use of information for behavior (Passingham and Sakai 2004), establishing response selection (Rowe et al. 2000) or a cognitive set (Rowe et al. 2007), spatial attention (Buschman and Miller 2007; Ikkai and Curtis 2011; Messinger et al. 2009), and behavioral rules (Wallis et al. 2001; White and Wise 1999). Spatial information processes are part of most of these cognitive functions and these cognitive functions cannot be separated from spatial information processes. Therefore, a detailed examination of the characteristics of prefrontal neural activity should provide important information regarding how spatial information is represented and how spatial information interacts with non-spatial information and participates in a variety of 
cognitive functions including working memory.

\section{Behavioral paradigms that examine the representation of space in the prefrontal cortex}

Since Jacobsen (1936) first reported that monkeys with bilateral lesions of the prefrontal cortex exhibited impairment in delayed-response performance, the delayed-response task had been widely used to examine spatial representations in the prefrontal cortex. This is because the construction and maintenance of the internal representation of spatial information (e.g., memory of the baiting position) are crucial for the correct performance of the delayed-response task and because the impairment of delayed-response performance by bilateral lesions of the lateral prefrontal cortex has been repeatedly confirmed by many lesion studies using monkeys (see Fuster 2008). Originally, hand-reaching behavior toward a baiting position or a response panel was used as the response behavior. However, saccadic eye movements are now often used as the response behavior. In addition to the delayed-response task, space representation and its function in the prefrontal cortex have been examined using a variety of behavioral tasks including delayed matching-to-position tasks, spatial attention tasks, sequential response tasks, spatial maze tasks, and delayed conditional response tasks.

\subsection{Delayed-response tasks}

The delayed-response task has been widely used to examine visuospatial representation in the prefrontal cortex. Originally, the delayed-response task was performed using the Wisconsin general test apparatus (WGTA) (Fig. 1). Hand-reaching behavior toward the baiting position was used as the response behavior. In the WGTA, the monkey is kept in a small cage in the laboratory. The cage is placed at one side of the experimental table. The experimenter sits at the other side of the table and presents 
cue stimuli and the reward at appropriate positions on the table. Usually, two baiting positions are prepared on the experimental table (see Fig. 1B), and the experimenter puts the reward in either position. An opaque screen is placed in front of the cage and the experimenter winds the screen up or down. After an inter-trial interval of several seconds, the opaque screen is raised. The experimenter puts the reward on the table at either the right or left baiting position (cue period) and then covers both baiting positions with identical opaque plates while the monkey is watching the experimenter's behavior. After these procedures, the opaque screen is lowered and an enforced delay of several seconds or minutes is introduced (delay period). At the end of the delay period, the opaque screen is raised and the monkey is now allowed to select the position where the reward has been hidden (response period). If the monkey selects the correct baiting position, it receives the reward. If the monkey selects the wrong position, the opaque screen is immediately lowered and an additional time-out period of several seconds or minutes is introduced.

Since the two baiting positions are covered with identical opaque plates, no external clue is present to inform the subject where the reward is hidden. The question where the reward is hidden can only be solved by memory of the baiting position. The subject is required to internally maintain the information where the reward was placed during the delay period and to use this maintained spatial information to guide a correct selection. Thus, to perform the delayed-response task correctly, the subject needs to construct an internal representation of spatial information, maintain this representation during the delay period, and then use it to perform a correct response. Therefore, the delayed-response task is a behavioral task that is able to examine the internal representation of spatial information.

Jacobsen (1936) first reported that a large bilateral lesion of the lateral prefrontal cortex caused permanent impairment of delayed-response performance in monkeys. Subsequent lesion studies using monkeys have repeatedly confirmed Jacobsen's 
original observation. These studies clearly showed that the lateral prefrontal cortex is critical for performance of the delayed-response task (see Fuster (2008)). Although Jacobsen (1936) initially made a large bilateral lesion in the lateral prefrontal cortex, subsequent studies tried to localize the critical area for this impairment within the prefrontal cortex. Butters et al. (1971) showed that the middle one-third of the principal sulcal area is critical for correct performance of the delayed-response task, since bilateral lesions of this localized area produced severe impairment in delayed-response performance. Rosenkilde (1979) summarized the results of lesion studies using monkeys and indicated that the cortex within and surrounding the principal sulcus is critical for performing the delayed-response task and the spatial delayed alternation task, both of which require the internal representation of spatial information. However, delayed matching-to-sample tasks and delayed non-matching-to-sample tasks, both of which require the internal representation of non-spatial information (e.g., object, color, shape), were not impaired by lesions in this area.

In addition to animal studies, Freedman and Oscar-Berman (1986) first showed that human patients with bilateral frontal lobe damage exhibited the selective impairment of delayed-response performance. Recent functional brain imaging studies have also revealed significant activation in the lateral prefrontal cortex while human subjects performed delayed-response tasks or tasks that required the temporary maintenance of spatial information (Baker et al. 1996; Brown et al. 2004; Curtis et al. 2004; Curtis and D’Esposito 2006; D’Esposito et al. 2000; Postle et al. 2000a).

Thus, the delayed-response task is a suitable task to examine spatial representation in the prefrontal cortex. Since delayed-response performance is closely linked to the functions of the lateral prefrontal cortex, this cortical area must include unique mechanisms to maintain and process spatial information necessary to perform the delayed-response task. This is why the delayed-response task and the spatial delayed 
alternation task have been widely used to examine the neural mechanisms of spatial representation in the prefrontal cortex.

\subsection{Oculomotor version of the delayed-response task (ODR task)}

For the neurophysiological examination of spatial representation in the prefrontal cortex, Fuster and Alexander (1971), Fuster (1973), Kubota et al. (1974), Niki (1974a), Niki and Watanabe (1976), and Kojima and Goldman-Rakic (1982, 1984) have used manual versions of the delayed-response task, and Kubota and Niki (1971) and Niki (1974b, 1974c) have used manual versions of the delayed alternation task. Recently, an oculomotor version of the delayed-response task (oculomotor delayed-response task or ODR task) has been used to examine the processing of spatial information in the prefrontal cortex (Constantinidis et al. 2001a, 2001b; Funahashi and Inoue 2000; Funahashi et al. 1989, 1990, 1991, 1993a, b; Inoue and Funahashi 2002; Joseph and Barone 1987; Meyer et al. 2011; Rainer et al. 1998b; Rao et al. 1997; Sawaguchi 1998; Sawaguchi and Iba 2001; Takeda and Funahashi 2002, 2004, 2007; Watanabe et al. 2006; Watanabe and Funahashi 2007; Wilson et al. 1993).

The ODR task consists of a memory-guided saccade task, which was developed and extensively used for oculomotor studies (Bruce and Goldberg 1985; Hikosaka and Wurtz 1983; Izawa et al. 2004; Kastner et al. 2007; Kojima et al. 2010; Pertzov et al. 2011). In the ODR task (Fig. 2A), the monkey usually faces a computer monitor on which a fixation target (FT, a small spot) and visual cues are presented. The monkey's eye positions are continuously monitored (e.g., by a magnetic search coil technique). After an inter-trial interval of several seconds, the FT is presented at the center of the computer monitor. If the monkey looks at the FT for a certain period (e.g., $1 \mathrm{~s}$, fixation period), a visual cue is presented briefly (e.g., $0.5 \mathrm{~s}$, cue period) at one of several predetermined peripheral positions around the FT. The monkey is required to maintain fixation on the FT throughout the cue period and the subsequent delay period (e.g., $3 \mathrm{~s}$ ). 
At the end of the delay period, the FT is extinguished. This is the Go signal for the monkey to make a saccade within the maximum response period (e.g., $0.5 \mathrm{~s}$ ) to the position where the visual cue had been presented. If the monkey makes a correct saccade, a drop of liquid reward is given.

The ODR task offers several advantages over the ordinary manual delayed-response task. First, in the ODR task, visual cues can be presented at multiple locations. For example, Funahashi et al. (1989) and Constantinidis et al. (2001b) used 8 cue positions, Takeda and Funahashi (2002) used both 4 and 8 cue positions, Sawaguchi and Yamane (1999) used 4 cue positions, and Sawaguchi and Iba (2001) used 16 cue positions. In contrast, in the manual delayed-response task, only two positions (usually right and left positions) had been used as cue positions (Fuster 1973; Kubota et al. 1974; Niki 1974a; Niki and Watanabe 1976). Second, since the monkey is required to maintain fixation on the FT during the delay period, unnecessary gross movements or behavior can be suppressed during the delay period. Especially, gazing at the cue position during the delay period can be inhibited. This is important because no spatial memory is required if the monkey continues to gaze at the cue position during the delay period. In addition, gaze-related activity has been observed in prefrontal neurons (Suzuki and Azuma 1977; Suzuki et al. 1979). These neurons exhibit tonic sustained excitation while the monkey continues to gaze at a visual target. In addition, the magnitude of this activation was modulated by the difference in the gaze direction. This effect has been called an angle-of-gaze effect and was also observed in parietal neurons (Andersen and Mountcastle 1983; Andersen et al. 1985b; Galletti and Battaglini 1989). Therefore, when spatial memory-related activity is examined in the prefrontal cortex, gaze-related effects should be removed from the activity during the delay period. In addition, unnecessary eye movements during the delay period should also be inhibited since many prefrontal neurons exhibit saccade-related activity (Boch and Goldberg 1989; Funahashi et al. 1991). Third, we can obtain trajectories of saccades and the 
spatial distribution of the ends of saccades for each cue condition. Using this saccade-related behavioral data, we can quantitatively estimate how well the monkey maintained spatial information during the delay period and then used it for its behavioral responses (e.g., Funahashi et al. 1993a). Fourth, the visual cue is presented briefly only during the cue period and no clue for performing correct saccades is presented during either the delay or response periods. Therefore, the maintenance of the spatial representation of the visual cue during the delay period is crucial for correct performance. Fifth, since visuospatial information is eventually transformed into motor information to perform saccades in the ODR task, we can examine how sensory- or motor-related spatial information is encoded and processed in prefrontal neurons (e.g., Funahashi et al. 1993b; Funahashi and Inoue 2000; Takeda and Funahashi 2002, 2004, 2007).

Thus, the ODR task must be an appropriate paradigm to examine spatial representation in the prefrontal cortex in detail. Using the ODR task, Funahashi et al. (1993a) showed that unilateral lesions restricted to the cortex within and surrounding the principal sulcus produce mnemonic "scotomas" in monkeys. They observed an impairment of memory-guided saccade performance only when the visual cues were presented in the visual field contralateral to the hemisphere with lesions. The severity of the impairment of memory-guided saccade performance increased as the length of the delay period increased. Most of errors were incorrect saccades directed toward wrong directions during the response period, and not the errors by breaking fixation during the delay period. However, no impairment was observed when the visual cues were presented in the visual field ipsilateral to the hemisphere with lesions. And, no impairment was observed when monkeys performed visually guided saccades toward any direction including contralateral visual field. These results indicate that the lateral prefrontal cortex, especially the cortex within and surrounding the principal sulcus, is crucial in spatial mnemonic functions and that using the ODR task is the best available 
paradigm to examine the nature of space representation and neural mechanisms of spatial information processing in the prefrontal cortex in detail. Therefore, an analysis of prefrontal neural activity during ODR performance could provide important evidences to understand how spatial information is represented and processed in the prefrontal cortex.

\subsection{Task-related prefrontal activities observed during ODR performance}

To understand spatial information processing, several important task-related activities have been observed in prefrontal neurons while monkeys performed the ODR task. For example, cue-period activity is a phasic excitatory response that occurs after presentation of the visual cue (Funahashi et al. 1990; Takeda and Funahashi 2002). An example of cue-period activity is shown in Fig. 2B. Most cue-period activity exhibited spatial selectivity, in that cue-period activity was observed only when the visual cues were presented within a certain area in the visual field (Funahashi et al. 1990). A response similar to cue-period activity was also observed in a visual probe task, in which visual stimuli were presented at the same positions as in the ODR task, but had no behavioral significance (Funahashi et al. 1990). Therefore, cue-period activity must be a visual response to the presentation of the visual cue. Prefrontal neurons have been shown to respond to simple visual stimuli (e.g., spot or bar stimuli) and to have visual receptive fields (Mikami et al. 1982; Suzuki and Azuma 1983). Therefore, an analysis of the spatial characteristics of cue-period activity could provide important information for understanding the characteristics of spatial representation in the prefrontal cortex.

Phasic excitatory activity has also been observed during the response period (Funahashi et al. 1991; Takeda and Funahashi 2002). An example of response-period activity is shown in Fig. 2B. Response-period activity can be classified into two types based on whether it started before or after the initiation of the saccadic eye movement (pre-saccadic or post-saccadic activity, respectively). In the prefrontal cortex, most 
neurons exhibit post-saccadic activity, while a minority exhibits pre-saccadic activity (Funahashi et al. 1991; Takeda and Funahashi 2002). Since post-saccadic activity begins after the initiation of saccadic eye movement, this activity does not participate in the initiation, execution, or control of saccadic eye movements. However, almost all post-saccadic activities and all pre-saccadic activities show selectivity regarding the saccade direction. Thus, the directional selectivity of response-period activity also provides important information for understanding the characteristics of spatial representation in the prefrontal cortex.

An important task-related activity that is observed in prefrontal neurons during ODR performance is delay-period activity. An example of delay-period activity is shown in Fig. 2B. Most delay-period activity is tonic sustained activation during the delay period. Delay-period activity was usually initiated several-hundred-ms after the presentation of the visual cue, was tonically maintained during the delay period, and ended just after the saccade was initiated (Funahashi et al. 1989; Goldman-Rakic et al. 1990). Although no visual stimulus was presented except for the FT and the monkey only maintained gazing at the FT during the delay period, most delay-period activity exhibited directional selectivity, in that delay-period activity was only observed in trials when the visual cue was presented at a certain area in the visual field (Funahashi et al. 1989; Funahashi and Takeda 2002). The internal maintenance of spatial information regarding where the visual cue was presented is crucial for performing correct eye movements during the response period. Therefore, delay-period activity is thought to be a neural correlate for maintaining an internal representation of spatial information (Funahashi 2001; Funahashi and Kubota 1994; Goldman-Rakic 1987). Thus, an analysis of the spatial characteristics of delay-period activity should provide essential information regarding the maintenance of an internal representation and the processing of spatial information in the prefrontal cortex. 
Thus, the ODR task is an important behavioral task for studies on the spatial representation in the prefrontal cortex. An examination of the spatial characteristics of task-related activity observed during ODR performance should provide important evidence for understanding the representation and processing of spatial information in the prefrontal cortex.

\subsection{Navigation tasks}

When we travel within a city and want to go to a particular place (e.g., a museum), our spatial knowledge about the city plays a critical role in navigation. Several studies using human subjects have indicated that the prefrontal cortex contributes to spatial navigation (Ciaramelli 2008; Spiers 2008, Ma et al. 2012; Yoshida and Ishii 2006). For example, Ciaramelli (2008) reported the navigation behavior of a patient who had ventromedial prefrontal damage. The patient showed severe difficulty in way-finding in his home town, although he had intact knowledge of landmarks and routes in this town. The patient had no problem in remembering his spatial goal, but often lost his way in getting to the goal by being attracted to familiar locations. Yoshida and Ishii (2006) used a spatial navigation task on a computer screen and observed the activation of the bilateral anterior prefrontal cortex using fMRI.

Mushiake et al. (2001) developed a path-finding task using an artificial maze presented on a computer monitor (Fig. 3). They asked monkeys to move a cursor on the monitor and to find an appropriate path to get to the goal on the maze. To perform this task, the monkeys initially needed to learn an association between the direction of the cursor movement and the direction of wrist rotation (supination or pronation). After the monkeys learned this association, they were informed of both a start position and a goal position on the maze. The monkeys were required to find the path to get to the goal by using the cursor by rotating both wrists appropriately. They found that monkeys always took the shortest path to get to the goal. They also found that monkeys changed the path 
in a flexible and adaptive manner when the experimenter put distracters on the way to the goal. Mushiake et al. (2006) examined prefrontal activities while monkeys performed a path-finding task and found that prefrontal neurons encoded each of all forthcoming directions of cursor movements, but not wrist movements, during the preparation period (before the initiation of the first cursor movement). In this task, monkeys usually used three cursor movements to reach the goal. The authors found three types of prefrontal neurons, the activities of which encoded the directions of the first, second, or third cursor movement during the preparation period, respectively. They also examined the activities of motor cortex neurons and found that all motor cortex neurons encoded the directions of wrist movements, rather than the directions of cursor movements. Further, Saito et al. (2005) found two kinds of delay-period activity, which were observed between the presentation of the goal and the initiation of cursor movement. One type of delay-period activity encoded the position of the immediate goal, where the monkey needed to move the cursor as the first step, while the other type of delay-period activity encoded the position of the final goal. Using the same navigation task, Mushiake et al. (2002) observed prefrontal activation in human subjects by event-related fMRI studies. Thus, these results show that prefrontal activities represent spatial information necessary to navigate through the maze and that different prefrontal neurons encode different kinds of spatial information.

Genovesio et al. (2006) observed results similar to those of Saito et al. (2005), although their task was not a navigation task. In their task, the monkeys needed to select one of three spatial goals (future goal) based on the combination of a symbolic visual cue that had appeared in the previous trial and the previous goal that the monkey selected. When a symbolic visual cue presented at the fixation target was the same as in the previous trial, the monkey was required to use the previous goal as the future goal. When the visual cue changed, the monkey needed to select one of the two remaining positions as the future goal. They found that prefrontal neurons encoded the positions of 
either the previous goal or the future goal and that a few neurons encoded both positions. These results suggest that separate prefrontal networks encode spatial information for each sub-goal and the final goal and that these networks are not directly linked to motor behavior. Thus, by using a rather complex spatial task such as an artificial maze and asking monkeys to find an immediate goal or a final goal in space, we can analyze the characteristics and functions of spatial representation in the prefrontal cortex.

\subsection{Spatial sequencing response tasks}

The dorsolateral prefrontal cortex has been implicated in procedural sequence learning in human functional imaging studies (Jenkins et al. 1994; Robertson et al. 2001). Robertson et al. (2001) used serial reaction time tasks, in which either the position, the color, or the combination of position and color of the stimulus cued the response. They examined the effects of repetitive transcranial magnetic stimulations (TMS) applied to the dorsolateral prefrontal cortex during task performance under the position condition, the color condition, or the combined condition. Repetitive TMS prevented the learning of sequential performance only in the position condition, and had little effect in the color and combined conditions. In addition, no effect was observed in any condition when repetitive TMS was applied to the parietal cortex. Thus, these results indicate that the prefrontal cortex plays an important role in sequence learning based on spatial information.

Single-neuron activity in the prefrontal cortex has been analyzed while monkeys performed sequential behaviors. Barone and Joseph (1989) examined prefrontal activity while monkeys performed a task that included spatial sequencing responses by saccadic eye movements and hand-reaching behaviors. In their task, three targets were illuminated in random order and the monkey needed to press each target in the same order as their illumination. They found that prefrontal neurons encoded the spatial position of a particular target as well as the temporal order of its illumination. 
Funahashi et al. (1993c, 1997) also examined prefrontal activities while monkeys performed a spatial sequencing task. In their task, monkeys were required to perform sequential hand-reaching responses to two of three targets in the same temporal order as that of target presentation during the cue period. They examined prefrontal activity during the delay period and found two groups of activities. One group exhibited spatially selective activity, such that delay-period activity was observed only when either target was presented at a particular position. The other group exhibited pair-dependent activity, such that delay-period activity was observed only when both targets were presented at particular positions. Interestingly, most of these two groups of neurons exhibited delay-period activity depending on the temporal order of target presentation. In these neurons, delay-period activity was observed only when the two targets were presented, for example, at the right and middle positions in the order. They concluded that these delay-period activities could contribute to simultaneously maintain multiple spatial information. Delay-period activity representing the temporal order of stimulus presentation has been reported in the prefrontal cortex by Ninokura et al. (2003, 2004), although they used non-spatial object stimuli. The combined representation of spatial and temporal information regarding a particular stimulus would be an effective method for a single prefrontal neuron to participate in planning a complex behavior. The fact that activity representing a sub-goal or the final goal was observed in the prefrontal cortex could support this notion.

\section{Space representation in the prefrontal cortex}

\subsection{Functional heterogeneity in the prefrontal cortex}

Evidence that the prefrontal cortex plays a critical role in representing and processing spatial information has been demonstrated by a variety of experiments. With regard to behavioral studies, Rosenkilde (1979) summarized the functional 
heterogeneity of the monkey prefrontal cortex and indicated that it contains 5 sub-regions (principal sulcal area, superior prefrontal convexity, inferior prefrontal convexity, medial orbital cortex, and arcuate concavity). Among these sub-regions, lesions of the principal sulcal area produce impairment in only two tasks: the spatial delayed-response task and spatial delayed alternation task, while lesions of the inferior prefrontal convexity produce impairment in a delayed matching-to-sample task, a go/no-go discrimination task, and a delayed object alternation task, most of which require non-spatial information (difference in color, shape, texture, or object). Although large bilateral lesions of the prefrontal cortex produced severe impairment in the delayed-response task, Butters et al. (1971) showed that lesions of only the middle one-third of the principal sulcus is sufficient to produce impairment in the delayed-response task. Thus, behavioral studies have indicated that the prefrontal cortex, especially the cortex within and surrounding the principal sulcus, plays an important role in the processing of spatial information.

The notion that the cortex surrounding the principal sulcus plays an important role in the processing of spatial information was also supported by the results of anatomical studies. Ungerleider and Mishkin (1982) proposed two visual pathways in the cerebral cortex. The dorsal visual pathway includes the posterior parietal cortex and plays a significant role in the processing of spatial aspects of visual information (position, movement, etc.), while the ventral visual pathway includes the inferior temporal cortex and plays a significant role in the processing of non-spatial aspects of visual information (color, shape, texture, etc.). Anatomical studies have shown that the cortex within and surrounding the principal sulcus receives major inputs from the parietal cortex (Andersen et al. 1985a; Cavada and Goldman-Rakic 1989), while the ventral convexity of the prefrontal cortex receives inputs mainly from the temporal cortex (Petrides and Pandya 1994; Seltzer and Pandya 1989). Thus, anatomical studies suggest that functionally different sectors are present in the lateral prefrontal cortex; the 
cortex within and surrounding the principal sulcus mainly receives information from the dorsal pathway and participates more in visuospatial information processing, while the ventral convexity mainly receives information from the ventral pathway and participates more in non-spatial visual information processing.

Prefrontal neurons are known to respond to a variety of sensory stimuli. By using visual, auditory, and somatosensory stimuli, Tanila et al. (1992) examined the sensory modalities that each prefrontal neuron responded to while monkeys were sitting quietly in a monkey chair. Among dorsolateral prefrontal neurons, 29\% responded to visual stimuli, $2 \%$ responded to auditory stimuli, $7 \%$ responded to somatosensory stimuli, and only 9\% responded to more than one sensory modality. Tanila et al. (1993) also examined sensory responses of single neurons and their locations in the prefrontal cortex. Visual neurons were distributed widely in areas 46 and 9. Somatosensory neurons were concentrated in the middle third of the inferior bank of the principal sulcus. Oculomotor neurons were found caudally in both banks of the principal sulcus.

These results indicate that, although the prefrontal cortex receives a variety of sensory information from the posterior cortices, the prefrontal cortex may be functionally compartmentalized with respect to the nature of its sensory inputs. Regional differences in the processing of spatial and non-spatial information within the lateral prefrontal cortex have been proposed. For example, Goldman-Rakic proposed that the mid-dorsolateral prefrontal cortex participates mainly in the processing of visuospatial information, whereas the mid-ventrolateral prefrontal cortex participates in the processing of non-spatial visual information (Goldman-Rakic 1996a, 1996b, 1998; Levy and Goldman-Rakic 2000). Her proposal has been supported by the anatomical studies described above, neurophysiological studies (Meyer et al. 2011; O Scalaidhe et al. 1997, 1999; Wilson et al. 1993), lesion studies using monkeys (Levy and Goldman-Rakic 1999), and functional brain imaging studies (Courtney et al. 1996, 1998; Glahn et al. 2002; Leung et al. 2002; McCarthy et al. 1994; Mottaghy et al. 2002; 
Sala et al. 2003). However, neurons encoding spatial information and non-spatial information can be observed in both mid-dorsolateral and mid-ventrolateral prefrontal areas (Quintana et al. 1988; Rao et al. 1997; Sakagami and Niki 1994). Many neurons exhibit task-related activity in both spatial and non-spatial working memory tasks (Rao et al. 1997). Rizzuto et al. (2005) showed spatial selectivity in the human ventrolateral prefrontal cortex. Therefore, although the prefrontal cortex may be functionally compartmentalized with respect to the nature of its sensory inputs, spatial and non-spatial information would be mixed up by extensive interactions among neurons. Each prefrontal neuron could represent not just one modality of information, but rather might represent a range of modalities of information depending on the demands of the task. This notion is supported by functional brain imaging studies (Nystron et al. 2000; Owen et al. 1998, 1999; Postle et al. 2000b; Rushworth et al. 1997).

\subsection{Characteristics of visual responses in the prefrontal cortex}

Visual responses are frequently observed in prefrontal neurons. These responses can be thought to represent physical properties of visual stimulus (color, shape, position, brightness, texture, or moving direction). In fact, one group of prefrontal neurons respond to physical properties of a visual stimulus regardless of whether it is relevant or irrelevant or meaningful or meaningless for performing cognitive tasks, while another group of prefrontal neurons are activated only when visual stimuli are relevant for performing the task.

Visual responses are often observed while subjects perform a variety of behavioral tasks. In these tasks, subjects are instructed how to behave during the task by visual stimuli (visual cues). Therefore, not only the physical properties of the visual stimuli (color, shape, position, or moving direction), but also the behavioral meaning of the stimuli in the task could be represented in these visual responses. In fact, several studies have shown that a large number of prefrontal neurons exhibited significant 
responses to visual cues and that a large proportion of these activities did not represent physical properties of the visual cues, but rather represented the behavioral meaning of the visual cues, such as response directions (e.g., right or left) or response types (e.g., Go or No-Go response) (Sakagami and Niki 1994; Sakagami and Tsutsui 1999; Watanabe 1986a, 1990).

On the other hand, it has also been shown that the responses to the visual cues observed in cognitive tasks were similar to those observed in visual control tasks, in which the visual stimuli had no behavioral significance (Funahashi et al. 1990; Lauwereyns et al. 2001). Funahashi et al. (1990) examined cue-period activity observed during the ODR task. Most of the cue-period activity exhibited directional selectivity, in that cue-period activity was observed only when the visual cue was presented at a certain direction from the fixation target. Most of the best directions of cue-period activity were directed toward the visual field contralateral to the hemisphere where the neurons were located. The response characteristics of cue-period activity in the ODR task were compared to the visual response in the visual probe task in the same prefrontal neuron. No difference was found in directional selectivity or response magnitude between cue-period activity and the visual response. Therefore, they concluded that cue-period activity observed in the ODR task is a visual response to the presentation of the visual cue. However, it is not clear whether cue-period activity represents only physical properties of the visual cue or also other-than-visual properties, such as motor information. Takeda and Funahashi (2002) used both the ODR task and an R-ODR task, in which subjects were required to make a saccade $90^{\circ}$ clockwise from the cue direction, and compared the best directions of cue-period activity recorded from the same neuron between these two tasks. If the best directions in these two tasks were the same, then cue-period activity would represent spatial information of the visual cue. However, if the best directions were different by $90^{\circ}$, then cue-period activity would represent information regarding the saccade direction. They found that all of the cue-period 
activities represented spatial information of the visual cue. Thus, cue-period activity observed in the ODR task is a visual response to the visual cue and this activity can be observed regardless of whether it is relevant or irrelevant for performing the task.

Lauwereyns et al. (2001) trained monkeys to perform a multidimenional Go/No-Go discrimination task, in which monkeys were required to discriminate either the color or the direction of motion of a visual target to make a correct hand movement. The essence of this task was that monkeys needed to extract relevant information (color in the color-discrimination condition and direction of motion in the motion-discrimination condition) while ignoring irrelevant information (direction of motion in the color-discrimination condition and color in the motion-discrimination condition). They found that around $40 \%$ of prefrontal activities were affected by task-irrelevant visual features and that the responses to an irrelevant feature were positively correlated with the responses to the same feature when it was relevant.

Thus, although a large number of prefrontal neurons receive visuospatial information from posterior association cortices, a hierarchical organization of information processing might be present among prefrontal neurons. A population of neurons might receive sensory inputs from posterior cortices, while another population of neurons might act as integrators to combine and integrate sensory inputs with other input. This might be why some neurons exhibit visual responses regardless of whether the visual stimulus is relevant or not for performing cognitive tasks, while the visual responses of other neurons are strongly affected by the relevance for performing behavioral tasks.

\subsection{Topographic visual map in the prefrontal cortex}

A large number of prefrontal neurons showed transient excitation or inhibition when the visual stimulus was presented in the visual field. Although it is well known that the frontal eye fields receive visual inputs from posterior visual areas, neurons in 
the prefrontal cortex other than the frontal eye fields are also known to respond to visual stimuli (Funahashi et al. 1990; Ito 1982; Kubota et al. 1974; Mikami et al. 1982; Pigarev et al. 1979; Rizzolatti et al. 1981; Suzuki and Azuma 1983). Some prefrontal neurons respond to rather complex visual features, such as natural objects or faces $(\mathrm{O}$ Scalaidhe et al. 1997; Pigarev et al. 1979; Wilson et al. 1993) or body parts in the extrapersonal space (Rizzolatti et al. 1981), while other neurons respond to simpler visual stimuli such as a slit or a spot of light (Ito 1982; Mikami et al. 1982; Suzuki and Azuma 1983).

Prefrontal visual neurons have visual receptive fields in the visual field (Mikami et al. 1982; Suzuki and Azuma 1983). Mikami et al. (1982) examined prefrontal responses to a moving or stationary slit stimulus presented on a wide tangential screen while monkeys gazed at a central fixation target. Many prefrontal neurons exhibited transient excitatory responses to the presentation of visual stimuli. The visual receptive field varied in size from $10^{\circ} \times 10^{\circ}$ to $60^{\circ} \times 60^{\circ}$ and was located mainly in the contralateral visual field. Suzuki and Azuma (1983) showed topographic distributions of the eccentricity and size of visual receptive fields in prefrontal visual neurons (Fig. 4). Neurons that were located in the lateral prefrontal cortex and close to the inferior arcuate sulcus had smaller receptive fields and represented the foveal and parafoveal regions, while those located in the medial part of the prefrontal cortex had larger receptive fields and represented a more eccentric position from the fovea. The size of the receptive field gradually increased for anteriorly located neurons, and this tendency was observed without a change in the eccentricity of the center of the receptive field. Thus, the prefrontal cortex not only receives visual information from posterior association cortices but also exhibits a topographic distribution of visual neurons based on the size of the receptive field and the eccentricity of the receptive field center.

The presence of such a topographic distribution of prefrontal visual neurons 
suggests the presence of a spatial map in the prefrontal cortex. Recently, spatial maps based on retinotopic coordinates have been identified not only in the striate and pre-striate cortices (Arcaro et al. 2009; Engel et al. 1997; Gardner et al. 2008; Kolster et al. 2010; Reppas et al. 1997) but also in the posterior parietal cortex (Arcaro et al. 2011; Sereno et al. 2001; Silver et al. 2005) of human brains by fMRI studies. The presence of a visuospatial map in the lateral prefrontal cortex has also been demonstrated by human fMRI studies. Hagler and Sereno (2006) used the n-back task using images of human faces and asked subjects to judge whether there was a 2-back match to the present image with respect to either identity or location. They found that prefrontal cortical areas represent visual space in an orderly, reproducible, topographic manner. Kastner et al. (2007) also examined visuospatial maps in the human prefrontal cortex using a memory-guided saccade task with 8 or 12 target positions distributed around the fixation target. Their task was a spatial working-memory task, in which the subject needed to judge whether or not a test stimulus was presented at the same position as a cue position by pressing a button. They found two topographic maps in the frontal cortex; one was located in the superior branch of the precentral sulcus and the other was located in the caudal part of the inferior frontal sulcus. Interestingly, they reported that saccade directions and memorized locations were represented in multiple locations of the map. Suzuki and Azuma (1983) showed topographic representations of the size and eccentricity of visual receptive fields in prefrontal visual neurons and indicated that the representations interacted. Therefore, if the topographic representation of visual space is present in the prefrontal cortex, it would not consist of simple maps as in the posterior parietal cortex, but would be maps with rather complex shapes, with a large amount of overlap among maps, as indicated by Kastner et al. (2007).

\subsection{Interaction between spatial and non-spatial information in the prefrontal cortex}


Since the prefrontal cortex has reciprocal connections with the posterior parietal cortex (Andersen et al. 1985a; Cavada and Goldman-Rakic 1989; Kawamura and Naito 1984; Petrides and Pandya 1984; Schwartz and Goldman-Rakic 1984) and the inferior temporal cortex (Kawamura and Naito 1984; Petrides and Pandya 1994; Seltzer and Pandya 1989; Ungerleider et al. 1989), the characteristics of visual responses observed in prefrontal neurons should be similar to those observed in posterior parietal neurons (Mountcastle et al. 1975; Sakata et al. 1997) and inferior temporal neurons (Desimone et al. 1984; Gross et al. 1972). In fact, Chafee and Goldman-Rakic (1998) showed that the characteristics of task-related activity during ODR task performance were similar between prefrontal neurons and posterior parietal neurons.

However, prefrontal visual neurons often responded differently from visual neurons in the posterior cortices. Prefrontal neurons respond only to the behaviorally relevant visual cue, even though other visual stimuli are presented simultaneously with the visual cue. For example, Rainer et al. (1998b) used a delayed matching-to-sample and location task to examine whether or not prefrontal neurons encoded only behaviorally relevant information. In their task, three objects were simultaneously presented at three different positions during the sample period. Before monkeys started this task, they were taught the target object by an association between the reward and an object. After a $1.5 \mathrm{~s}$ delay period, the same three objects were presented with a different spatial arrangement as test stimuli. Monkeys were required to release the lever if the target object was presented at the same position. They found that the magnitude of prefrontal activity correlated only with the position of the target object and that the positions of irrelevant objects had little or no effect on prefrontal activity.

A similar observation was reported by Hasegawa et al. (2000). They used a visual search task with a delay, in which a visual target was presented with 5 distracters during the cue period and, after a brief delay, monkeys were required to make a saccade to the position where the visual target was presented. They observed strong responses 
only when the visual target was presented within the neuron's visual receptive field, and little or no response when the distracter was presented in the neuron's visual receptive field.

Further, Hoshi et al. (2000) examined prefrontal activity under two matching conditions (location-matching and shape-matching). They presented either a circle or a triangle as a sample cue at one of three positions. In the location-matching condition, either a circle or triangle was presented at all three positions in the choice period and monkeys were required to reach for the position where the sample cue had been presented. In the shape-matching condition, one circle and one triangle were presented at two of three positions in the choice period and monkeys were required to reach for the position where the same shape as the sample cue had been presented. They found that, among neurons that responded to the sample cue, $42 \%$ showed only position-selective activity, $11 \%$ showed only shape-selective activity, $21 \%$ were affected by both the position and shape of the sample cue, and the remaining $26 \%$ responded to the sample cues non-differentially.

Similar results were obtained by Rao et al. (1997). Among prefrontal neurons that exhibited delay activity depending on either the object or location or both, $7 \%$ showed delay activity tuned to the object, $41 \%$ showed delay activity tuned to the location, and the remaining 52\% showed delay activity tuned to both the object and the location. Thus, nearly $90 \%$ of prefrontal delay activity was affected by the spatial location of the target object.

Thus, all of these studies indicate that a large proportion of prefrontal activities are affected by spatial information. Although the prefrontal cortex receives both spatial and non-spatial visual information from the posterior cortices, spatial information seems to strongly influence prefrontal information processing. Since both spatial and non-spatial information are necessary to perform the task, we need to further examine the mechanism by which spatial and non-spatial information are integrated in the 
prefrontal cortex. In addition, these results show that, although prefrontal neurons receive a variety of sensory information from the posterior cortices, many prefrontal neurons encode and process only behaviorally relevant information. This suggests the presence of a neural mechanism that discriminates behaviorally relevant information from irrelevant distracters. We again need to elucidate how and where relevant information is discriminated from irrelevant information in the brain.

\subsection{Behavioral context-dependent visual responses}

A significant number of prefrontal neurons have been shown to exhibit visual responses. However, some of these responses do not represent physical features of the visual stimulus, and instead represent the behavioral context or behavioral meaning in the task. For example, Watanabe (1990) used a stimulus-reward association task, in which one of two pattern stimuli was associated with a reward. He found that the response to the stimulus was modulated only by its physical features in $7 \%$ of the neurons examined, while the response was modulated depending only on whether or not the visual stimulus was associated with a reward in $67 \%$ of the neurons. A similar observation has been reported by Sakagami and Niki (1994). They used three types of symmetrically rewarded Go/No-Go discrimination tasks, in which monkeys were required to make a correct response based on either the color, shape, or position of the visual cue. Among the 328 prefrontal neurons examined, 76\% responded differentially during presentation of the visual cue depending only on the difference in behavioral responses (either Go or No-Go response), and not on the difference in physical properties of the visual cues (color, shape, or position). Only $2 \%$ responded differentially depending on the difference in the physical properties of the visual cues. Further, Sakagami and Tsutsui (1999) used a Go/No-Go selective attention task. In this task, monkeys were required to make either Go (lever-pressing) or No-Go (lever-holding) responses depending on either the color or direction of motion of visual 
target stimuli (moving colored dots). In the color condition, monkeys needed to attend to the color of moving stimuli and ignore the direction of motion, whereas in the motion condition, monkeys needed to attend to the direction of motion of moving stimuli and ignore the color. They found three types of neurons. "Color and motion cells" (37\%) were differentially active during presentation of the visual target under both conditions and their activation depended only on the response type (Go or No-Go). Similarly, "color cells" (32\%) were differentially active during presentation of the visual target and their activation depended solely on the response type under the color condition. Finally, "motion cells" (15\%) were differentially active during presentation of the visual target and their activation depended solely on the response type under the motion condition. Thus, since these prefrontal neurons were activated when visual stimuli were presented, they are usually classified as visually responsive neurons. However, the activities of these neurons did not represent the physical properties of the target stimuli, and instead represented the type of the response (either a Go or No-Go response).

Thus, although the activities of many prefrontal neurons were affected by the physical properties of the visual stimulus, these neurons were also strongly affected by the behavioral meaning or the behavioral context that the visual stimulus represented. Behavioral meaning-dependent activation and context-dependent activation are important features that are exhibited by prefrontal neurons. Thus, the prefrontal cortex processes and integrates sensory information received from the posterior corticies together with other cognitive information and generates behaviorally important and relevant information. This feature is important when we consider prefrontal functions. However, this feature is not limited to prefrontal neurons. Gottlieb et al. (1998) found that some neurons in the area LIP responded to visual stimuli that were only relevant for behavioral performance. Since little is known about the neural mechanisms that underlie the integration and processing of multimodal information and how relevant information is discriminated from irrelevant information, further studies are needed to elucidate 
these mechanisms in the prefrontal cortex.

\subsection{Organization of the auditory receptive field}

The prefrontal cortex has been shown to receive inputs from the auditory cortex (Romanski et al. 1999a, 1999b). Several studies in macaque monkeys have shown that prefrontal neurons respond to auditory stimuli and that prefrontal auditory responses exhibit similar spatial selectivity as prefrontal visual responses (Azuma and Suzuki 1984; Ito 1982; Romanski and Goldman-Rakic 2001; Suzuki 1985; Vaadia et al. 1986). Using pure tones, Ito (1982) examined prefrontal responses to visual and auditory stimuli while monkeys performed a simple reaction time task. He found that, while many neurons exhibited phasic responses to these stimuli, only a few responded to both modalities. Vaadia et al. (1986) examined prefrontal activity under four task conditions: auditory localization task, auditory detection task, visual localization task, and passive listening condition. They used a noise burst as an auditory stimulus. Auditory neurons exhibited a contralateral bias in response tuning. These neurons were more active in the auditory discrimination or detection tasks compared with the passive listening condition. They also found bimodal neurons in the prefrontal cortex. These bimodal neurons exhibited similar spatial tuning in the auditory and visual localization conditions.

The modality specificity was also examined using the ODR task with visual and auditory cues. Kikuchi-Yorioka and Sawaguchi (2000) examined the modality specificity of delay-period activity using the ODR task performed under either a visual or an auditory condition. They found that, among neurons that exhibited directional delay-period activity, 53\% were classified as visual-specific neurons, $17 \%$ were auditory-specific neurons, and $17 \%$ were non-specific neurons. Since a majority of prefrontal neurons exhibited modality-specific delay-period activity, they suggested the presence of parallel processes for visual and auditory spatial working memory in the 
lateral prefrontal cortex. Although they did not compare the spatial tuning of directional delay-period activity between visual-specific neurons and auditory-specific neurons, they showed that there was no significant difference in the discriminability of spatial locations between these two groups of neurons, which suggests that the spatial tuning characteristics are similar for the processing of visual and auditory information in the prefrontal cortex.

The characteristics of spatial representation by prefrontal neurons have usually been examined under the condition in which the stimuli are presented on the frontal plane (e.g., a tangential screen or a TV monitor placed in front of the subject). Since we always receive visual information from the frontal plane, it is appropriate to perform vision studies under this condition. However, auditory stimuli arrive not only from the front but also from the sides and the back of the body. Azuma and Suzuki (1984) examined the spatial tuning of prefrontal auditory neurons using auditory stimuli placed around a monkey's head. They found a contralateral bias in response tuning. In addition, they found that most of the neurons exhibited a maximum response to sound coming from lateral or posterolateral directions. Prefrontal visual neurons located in the ventral part of the prefrontal cortex represented visual information presented in the central region of the contralateral visual field, while visual neurons located in the dorsal part of the prefrontal cortex represented visual information presented in the peripheral region of the visual field (Suzuki and Azuma 1983). The dorsal part of the prefrontal cortex also included auditory neurons with best tunings that were directed toward lateral or posterolateral directions. By comparing the topographic distributions of visual receptive fields in prefrontal visual neurons with the spatial tuning of prefrontal auditory neurons, Suzuki (1985) concluded that prefrontal visual and auditory neurons function together to obtain information raised in the entire contralateral space, such that the receptive fields of prefrontal auditory neurons cover the spatial area that is not covered by the receptive fields of prefrontal visual neurons. 
Recently, Romanski and her group used a variety of vocalization and non-vocalization stimuli and examined prefrontal responses to these auditory stimuli (Averbeck and Romanski 2006; Romanski and Averbeck 2009; Romanski and Goldman-Rakic 2001; Romanski et al. 2004). They found that auditory responsive neurons were located mainly in the ventrolateral part of the prefrontal cortex. Most of these neurons were responsive to complex sounds including species-specific vocalizations, human speech sounds, and environmental sounds. However, only a few neurons responded to pure tones or noise stimuli. Cohen et al. (2004) found that ventrolateral prefrontal neurons were modulated by both the spatial and non-spatial (vocalization) attributes of an auditory stimulus. Since most of these neurons responded to complex sounds such as vocalization, prefrontal auditory neurons might participate in vocal communication, and not just in discriminating between different auditory stimuli.

Thus, some prefrontal neurons represent only visual information, some represent only auditory information, and some represent both visual and auditory information. Sensory modality-specific representation is observed not only in responses to stimulus presentation but also in cognitive activity such as mnemonic activity (delay-period activity). Comparison of the spatial tuning in bimodal neurons reveals that there is some similarity in the spatial tuning of visual and auditory activities. Interestingly, Mazzoni et al. (1996) reported similar spatial tuning characteristics in both sensory and mnemonic activities of area LIP neurons in auditory and visual saccade tasks. Bushara et al. (1999) found that the posterior parietal region and the lateral prefrontal cortex are specialized for auditory spatial processing using positron emission tomography (PET) while human subjects performed auditory and visual spatial localization tasks. Multimodal interactions of visual and auditory spatial information might occur in both the prefrontal cortex and the posterior parietal cortex.

\section{Spatial representation and spatial working memory}




\subsection{Task-related single-neuron activity observed during spatial working memory performance}

Since Jacobsen (1936) first reported that monkeys with bilateral lesions of the prefrontal cortex showed impaired delayed-response performance, behavioral tasks that include a delay (e.g., delayed-response task, delayed alternation, delayed matching-to-sample task) have been widely used to examine prefrontal functions. In the delayed-response task, for example, the subject is required to maintain spatial information during an imposed delay period and then to use that information to make a correct choice during the response period. Once the subject makes a choice, regardless of whether it is a correct choice or incorrect choice, spatial information maintained during the delay period is no longer necessary, since the baiting position changes from trial to trial. Therefore, the subject needs to discard previous spatial information and prepare to acquire new information. Thus, the subject is required to temporarily maintain and process spatial information while they perform the delayed-response task correctly. The temporary maintaining of information and the processing of this information are two main functions of working memory. Lesion studies in primates have revealed that the cortex within and surrounding the principal sulcus is the brain area that is responsible for correct performance of the delayed-response task (see Rosenkilde 1979; Fuster 2008). Therefore, this prefrontal area has been thought to include neural mechanisms for working memory (Funahashi 2001; Funahashi and Kubota 1994; Fuster 2008; Goldman-Rakic 1987; Miller 2000; Miller and Cohen 2001; Petrides 1994). This idea suggests that an analysis of prefrontal neural activities may provide important evidence regarding the neural mechanisms of working memory, such as how neural activities represent a variety of information, how neurons hold information for the necessary period, and how information is processed.

The delayed-response task has been frequently used as a spatial working 
memory task in neurophysiological experiments. Kubota and Niki (1971) and Fuster and Alexander (1971) first reported single-neuron activity recorded from the dorsolateral prefrontal cortex. Kubota and Niki (1971) found neurons that exhibited task-related activity while monkeys performed a spatial delayed alternation task. They found neurons that responded transiently to presentation of the visual cue and neurons that were activated during motor responses. On the other hand, Fuster and Alexander (1971) and Fuster (1973) found memory-related single-neuron activity while monkeys performed a manual delayed-response task. This memory-related activity (delay-period activity) consisted of sustained excitation during the delay period. Fuster (1973) found that neurons with delay-period activity comprised a major group in the dorsolateral prefrontal cortex. Delay-period activity was not observed in trials without baits or in error trials. Therefore, he concluded that delay-period activity reflects a role of the prefrontal cortex in mnemonic processes.

After Fuster (1973) reported delay-period activity in the dorsolateral prefrontal cortex, many studies have been performed to examine the characteristics of delay-period activity using various versions of the delayed-response task (Chafee and Goldman-Rakic 1998; Funahashi et al. 1989, 1993b, 1997; Kojima and Goldman-Rakic 1982, 1984; Kubota and Funahashi 1982; Niki 1974a, 1974b, 1974c; Niki and Watanabe 1976; Procyk and Goldman-Rakic 2006; Quintana et al. 1988; Sawaguchi and Iba 2001; Wilson et al. 1993). The most important feature of delay-period activity is that this activity exhibits directional selectivity, in that delay-period activity is observed only when the visual cue is presented at either the right or the left position (e.g., Niki 1974a). The characteristics of delay-period activity will be discussed in a later section.

In addition to memory-related delay-period activity, activation during presentation of the visual cue (cue-period activity) and activation during motor performance (response-period activity) have also been observed in the prefrontal cortex during delayed-response performance. Response-period activity has been observed 
when manual responses were used for the response behavior (Funahashi 1983; Funahashi et al. 1997; Fuster et al. 1982; Hoshi and Tanji 2004; Hoshi et al. 1998, 2000; Kojima and Goldman-Rakic 1984; Kojima et al. 1981; Kubota and Funahashi 1982; Kubota and Komatsu 1985; Kubota and Niki 1971; Kubota et al. 1980; Niki and Watanabe 1976; Quintana et al. 1988; Rosenkilde et al. 1981; Sawaguchi 1987; Sawaguchi et al. 1989; Watanabe 1986b; Yajeya et al. 1988) as well as when oculomotor responses were used for the response behavior (Barone and Joseph 1989; Boch and Goldberg 1989; Funahashi et al. 1991, 1993b; Joseph and Barone 1987; Sawaguchi and Iba 2001; Takeda and Funahashi 2002, 2004, 2007; Watanabe and Funahashi 2007; Watanabe et al. 2006). Response-period activity has often been shown to exhibit directional selectivity (Boch and Goldberg 1989; Funahashi et al. 1991; Joseph and Barone 1987; Kubota and Funahashi 1982; Takeda and Funahashi 2002; Watanabe et al. 2006), i.e., activity occured only when the response movement was directed toward one or a few particular directions. The comparison of response-period activity observed in the prefrontal cortex and movement-related activity observed in the motor cortex revealed a close similarity with regard to directional selectivity and temporal pattern (Kubota and Funahashi 1982). Therefore, response-period activity observed in the prefrontal cortex had been thought to participate in the initiation or execution of the response behavior (Fuster 2008; Kubota and Funahashi 1982; Kubota et al. 1974). However, in studies using oculomotor behavior, a large number of prefrontal neurons were active during eye movements (Boch and Goldberg 1989; Funahashi et al. 1991; Joseph and Barone 1987; Takeda and Funahashi 2002) and, although both pre- and post-saccadic responses were observed in the prefrontal cortex, a great majority of saccade-related activity was post-saccadic (Funahashi et al. 1991; Joseph and Barone 1987; Takeda and Funahashi 2002). Since the initiation of eye movement usually precedes the initiation of the manual response, pre-movement activity observed in the prefrontal cortex might not be directly related to the initiation or 
execution of the manual behavior, and instead might be saccade-related activity. Nevertheless, both pre- and post-saccadic activities observed in the prefrontal cortex exhibited directional selectivity. Thus, activity observed in the response period of the delayed-response task also represents spatial information.

\subsection{Characteristics of delay-period activity}

Delay-period activity, most of which is tonic sustained activation during an imposed delay period, has been considered to be a neural correlate of the mechanism for temporarily maintaining information (Funahashi 2001; Funahashi and Kubota 1994; Funahashi and Takeda 2002; Fuster 2008; Goldman-Rakic 1987; Miller 2000; Miller and Cohen 2001). Neurophysiological studies with the delayed-response task have shown that many prefrontal neurons exhibit delay-period activity (Funahashi et al. 1989; Fuster 1973; Kojima and Goldman-Rakic 1982; Niki 1974; Niki and Watanabe 1976). Furthermore, the duration of delay-period activity was prolonged or shortened depending on the length of the delay period (Funahashi et al. 1989; Fuster 1973; Kojima and Goldman-Rakic 1982). Delay-period activity was observed only in correct trials, and was truncated or not observed in error trials (Funahashi et al. 1989; Fuster, 1973). These evidences support the idea that delay-period activity is a neural correlate of the mechanism for temporarily maintaining the information that is necessary to perform this task correctly.

A great majority of delay-period activity has been shown to exhibit directional selectivity (Funahashi et al. 1989), in that delay-period activity was observed only when a visual cue was presented at a particular area in the visual field. The preferred direction of directional delay-period activity can be estimated by constructing a tuning curve (Funahashi et al. 1989). The preferred direction of delay-period activity differs from neuron to neuron. However, most delay-period activity had a best response direction that was toward the visual field contralateral to the hemisphere where the neuron was 
located (Funahashi et al. 1989). The tuning width of delay-period activity also differed from neuron to neuron; some neurons exhibited sharp tuning and others exhibited broader tuning. Based on these observations, it has been proposed that neurons with directionally selective delay-period activity have mnemonic receptive fields (memory fields) in the visual field, in that visual neurons have visual receptive fields (Funahashi 2001; Funahashi and Kubota 1994; Funahashi and Takeda 2002; Funahashi et al. 1989; Goldman-Rakic 1996a, 1996b, 1998; Rainer et al. 1998). Thus, delay-period activity observed in prefrontal neurons is considered to be a neural correlate of the mechanism for temporarily maintaining information. Spatial information must be maintained during the delay period in the delayed-response task. Since a great majority of delay period activity exhibited directional selectivity, delay-period activity observed during ODR performance could be a neural correlate of the mechanism for maintaining an internal representation of spatial information.

\subsection{What information does delay-period activity represent?}

If delay-period activity is a neural correlate of the mechanism for temporarily maintaining information, what information does delay-period activity represent while monkeys perform a delayed-response task? To perform delayed-response trials correctly, information regarding where the visual cue was presented and where to make a response are both important. Which information does delay-period activity represent? Niki and Watanabe (1976) first examined whether delay-period activity represented visuospatial information or motor information. They used two spatial delayed-response tasks. Right and left visual cues were used in one task, and upward and downward visual cues were used in the other. They also used a conditional position task with a delay, in which monkeys were required to press the right (left) response key after the delay when the visual cue was presented at the upward (downward) position. They examined the delay-period activity of a single prefrontal neuron under these three task conditions. If a 
neuron exhibited delay-period activity only in trials in which the visual cue was presented at the upward (downward) position, they concluded that this neuron's delay-period activity represented the position of the visual cue. On the other hand, if a neuron exhibited delay-period activity in trials in which the visual cue was presented at the right (left) and upward (downward) positions, they concluded that this neuron's delay-period activity represented the direction of the response behavior. They found that $70 \%$ of directional delay-period activity represented the position of the visual cue, whereas the remaining $30 \%$ represented the direction of the response behavior.

Although Niki and Watanabe (1976) provided an important result to consider spatial mnemonic functions of the prefrontal cortex, they did not control the monkey's eye movements during the delay period. As was explained before, many prefrontal neurons exhibit eye movement-related activities (Boch and Goldberg 1989; Funahashi et al. 1991; Joseph and Barone 1987; Takeda and Funahashi 2002). Therefore, Funahashi et al. (1993b) performed a similar experiment under the condition where the monkey's eye movements were strictly controlled by requiring the monkey to gaze at the fixation target during the delay period. They used delayed pro- and anti-saccade tasks. In the delayed pro-saccade task, the monkey was required to make a memory-guided saccade toward the cue direction after the delay, whereas in the delayed anti-saccade task, the monkey was required to make a memory-guided saccade opposite the cue direction. The directional selectivity of delay-period activity recorded from the same prefrontal neuron in these two tasks was compared. The result showed that a great majority $(68 \%)$ of directional delay-period activity represented information regarding the direction of the visual cue, whereas a minority (25\%) represented information regarding the saccade direction.

Similar results were obtained by Takeda and Funahashi (2002). They used the ODR task and a rotatory ODR (R-ODR) task (Fig. 5). In the R-ODR task, monkeys were required to perform memory-guided saccades to the direction $90^{\circ}$ clockwise from 
the cue direction. They defined whether delay-period activity represented the direction of the visual cue or the direction of the saccade by comparing the peak directions of the tuning curves constructed using delay-period activity obtained from the two task conditions. Since tuning curves were always constructed based on the cue directions, if the peak directions of both tuning curves were the same, delay-period activity could be defined as representing the direction of the visual cue. However, if the peak direction of the tuning curve constructed under performance of the ODR task was $90^{\circ}$ different from that of the tuning curve constructed under performance of the R-ODR task, delay-period activity could be defined as representing the direction of the saccade.

Figure 6 shows an example of delay-period activity representing the direction of the visual cue. This neuron exhibited excitatory delay-period activity in the $270^{\circ}$ and $315^{\circ}$ trials of the ODR task and in the $270^{\circ}$ trial of the R-ODR task. In this naming scheme, the name of the trial (e.g., $270^{\circ}$ trial) is based on the cue direction: in the $270^{\circ}$ trial of the R-ODR task, the visual cue was presented at the $270^{\circ}$ position, while saccades were directed toward the $180^{\circ}$ direction. The lower figure in Fig. 6 shows the tuning curves of delay-period activity obtained from two task conditions. The peaks of both tuning curves are located at the same cue direction. Thus, this neuron exhibited delay-period activity when the visual cue was presented at the $270^{\circ}$ and $315^{\circ}$ directions, regardless of the direction of the saccade. Therefore, it can be concluded that this delay-period activity represents the direction of the visual cue, not the direction of the saccade.

Figure 7 shows an example of delay-period activity that represents the direction of the saccade. This neuron exhibited excitatory delay-period activity only in the $0^{\circ}$ trial of the ODR task and the $90^{\circ}$ trial of the R-ODR task. In the $90^{\circ}$ trial of the R-ODR task, the visual cue was presented at the $90^{\circ}$ direction, but the saccade was directed toward the $0^{\circ}$ direction. Thus, this neuron exhibited delay-period activity when the saccade was directed toward the $0^{\circ}$ direction, regardless of the direction of the visual cue. Therefore, 
it can be concluded that this delay-period activity represents the direction of the saccade.

The lower figure in Fig. 7 shows the distribution of differences in the peak directions of delay-period activity between two tasks for the prefrontal neurons examined. In this figure, values close to $0^{\circ}$ indicate that delay-period activity was observed when the visual cue was presented at the same or a close direction in these two task conditions. Therefore, the delay-period activity of these neurons represents the direction of the visual cue. On the other hand, values close to $90^{\circ}$ indicate that delay-period activity represents the direction of the saccade. Using this method, Takeda and Funahashi (2002) found that a large proportion (86\%) of directional delay-period activity represented information regarding the direction of the visual cue, whereas only a small proportion (13\%) represented information regarding the saccade direction.

Thus, these studies showed that the majority of directional delay-period activity represented information regarding where visual cues were presented. Since the preferred direction of delay-period activity differed from neuron to neuron, Funahashi et al. (1989) proposed that prefrontal neurons that exhibit directional delay-period activity have mnemonic receptive fields (memory fields) in the visual field. The presence of memory fields in prefrontal neurons was supported by Funahashi et al. (1993a), Inoue and Funahashi (2002), and Rainer et al. (1998). Thus, delay-period activity is a neural correlate of the mechanism for maintaining an internal representation of visuospatial information.

It has been shown that most delay-period activity represents visuospatial information, whereas a minority represents forthcoming motor information in prefrontal neurons. Genovesio et al. (2006) reported that separate neural populations of the prefrontal cortex appear to represent different kinds of information. They showed that different neural populations represented either a future spatial goal or a previous spatial goal. In addition, observations that prefrontal delay-period activity reflected visuospatial 
attributes more than motor attributes were also obtained in experiments using indirect methods (Constantinidis et al. 2001; di Pellegrino and Wise 1993; Sawaguchi and Yamane 1999). For example, Sawaguchi and Yamane (1999) examined delay-period activity using a delayed matching-to-space task, in which monkeys were required to make either a lever-holding response (No-Go response) or a lever-releasing response (Go response) depending on whether or not the spatial position of the matching stimulus was the same as that of the sample stimulus. They found that $90 \%$ of recorded prefrontal activities showed selectivity with regard to the position of the stimulus, but not to the type of the response (Go or No-Go). Constantinidis et al. (2001a) examined prefrontal delay-period activity using the ODR task with two visual cues. They presented two visual cues simultaneously, but changed the luminance of one of the visual cues. Monkeys were required to make a saccade to the direction where the brighter visual cue had been presented. They found that a large population of neurons maintained the visuospatial attributes of remembered stimuli throughout the delay period.

On the other hand, Fukushima et al. (2004) showed that most delay-period activity observed in the prefrontal cortex encoded the direction of the saccade when monkeys performed an ODR-like task but were required to update the position of the saccade target sequentially through the presentation of non-spatial target-shift cues. They found neurons that were selectively activated when a particular saccade target was assigned by the target-shift cue. Although most delay-period activity represented visuospatial information when monkeys performed the ODR-like task, the proportion of delay-period activity that represents visuospatial information may change depending on the task demands. If visual information plays a major role in performance of the task (e.g., the ODR and R-ODR tasks), prefrontal neurons tend to maintain visuospatial information as directional delay-period activity. However, if motor information plays a major role in performance of the task, prefrontal neurons seem to more frequently 
represent motor information as directional delay-period activity. Thus, the information that delay-period activity represents may change flexibly depending on the demands of the task.

In summary, directional delay-period activity can be considered a neural correlate of the mechanisms for maintaining an internal representation of visuospatial information. Since delay-period activity can be observed in a variety of behavioral tasks and since information needed to represent is different from task to task, delay-period activity can temporarily represent a variety of information including visuospatial information, motor information, non-spatial visual features, quality differences in a single stimulus modality, or task rules. The type of information that is maintained by delay-period activity seems to change flexibly depending on the demands of the task. Visual information and motor information both contain spatial components (e.g., direction of the response in space). Therefore, directional delay-period activity that is observed during spatial working memory tasks should be an important topic for understanding how prefrontal activity represents spatial information and how this representation is used in the processing of spatial information in the prefrontal cortex.

\subsection{What coordinate system is used in directional delay-period activity?}

Although experimental results have shown that most directional delay-period activity represents the direction of the visual cue during delayed-response performance, the coordinate system that is used for the directional selectivity of delay-period activity is not clear. Niki (1974c) was the first to perform an experiment on this subject. He trained monkeys to perform a delayed alternation task using 4 horizontally arranged cue positions. In each trial, two cue positions were selected from among the 4 positions. Therefore, a particular cue position could be the right-side cue or the left-side cue depending on which other cue position was selected. In particular, either of the two cues located in the middle became either the right cue or the left cue depending on whether 
the leftmost cue or the rightmost cue was selected as the other cue position. He examined the selectivity of delay-period activity using these procedures and found that most delay-period activity encoded the relative spatial position of the visual cue. However, at the same time, the magnitude of delay-period activity was affected by the positions at which the visual cues were located on the response panel: although the relative spatial position of the visual cue had the greatest effect on the magnitude of delay-period activity, the difference in the position of the visual cue on the panel also affected the magnitude of delay-period activity.

The coordinate system that is used in the directional selectivity of delay-period activity was also examined using the ODR task with 4 visual cue positions under three fixation conditions (Funahashi and Takeda 2002). Three central fixation targets were arranged horizontally and separated by a visual angle of $10^{\circ}$ (Fig. 8A). Each fixation target had four peripheral cues (left, right, up, and down) at an eccentricity of $10^{\circ}$. Therefore, in this task, the right visual cue in the left fixation condition was presented at the same position as the left visual cue in the central fixation condition. And, the right visual cue in the central fixation condition was presented at the same position as the left visual cue in the right fixation condition. The fixation target that appeared and the visual cue that was presented were randomized in any given trial.

Figure $8 \mathrm{~B}$ shows examples of delay-period activity observed under the three fixation conditions. This neuron exhibited significant delay-period activity when the visual cue was presented on the left side of the fixation target. Significant delay-period activity was observed when the visual cue was presented on the left side in the right fixation condition, but no delay-period activity was observed when the visual cue was presented on the right side in the central fixation condition. Similarly, significant delay-period activity was observed when the visual cue was presented on the left side in the central fixation condition, but no delay-period activity was observed when the visual cue was presented on the right side in the left fixation condition. In the right fixation 
condition, the left visual cue was presented at the same spatial position as the right visual cue in the central fixation condition. Similarly, in the central fixation condition, the left visual cue was presented at the same spatial position as the right visual cue in the left fixation condition. Thus, this result indicates that this directional delay-period activity does not encode the position where the visual cue is presented on the monitor, but rather encodes the position relative to that of the fixation target. However, at the same time, the magnitude of delay-period activity was affected by the position of the fixation target on the monitor. As shown in Fig. 8B, delay-period activity had a greater magnitude when the fixation target was located at the center position compared the left position. This effect resembled an "angle-of-gaze" effect observed in posterior cortical areas (Andersen et al. 1985b; Andersen and Mountcastle 1983; Boussaoud 1995; Boussaoud et al. 1998; Galletti and Battaglini 1989; Rosenbluth and Allman 2002). The magnitude of delay-period activity was affected by the gaze position. However, the best cue position was the same across different gazing positions.

These results indicate that delay-period activity encodes the position of the visual cue relative to that of the fixation target, and not the position on the monitor. These results also indicate that the magnitude of delay-period activity is affected by the gaze angle. This indicates that the absolute spatial position of the fixation target plays an important role in encoding the relative position of the visual stimulus. Recently, Genovesio et al. (2011) showed that prefrontal activity represents relative distance. They presented two visual stimuli at different distances from the reference point and asked monkeys to identify the stimulus that was farther from the reference point. Although some neurons encoded the absolute distance of the stimulus from the reference point, a majority encoded the relative distance. Thus, their results are similar to those described above.

A variety of coordinate systems have been reported to encode visual stimuli. These include head-centered coordinate (Andersen et al. 1985b; Galletti et al. 1993; 
Kurata 2007; O'Dhaniel et al. 2005; Zhang et al. 2004), body-centerned coordinate (Beurze et al. 2010; McIntyre et al. 1998; Schlicht and Schrater 2007), object-centered coordinate (Olson and Gettner 1995, 1999; Olson and Tremblay 2000), body parts-centered coordinate (Fogassi et al. 1996; Graziano et al. 1997), viewer-centered coordinate (Carrozzo et al. 1999; McIntyre et al. 1997), and spatial coordinate (Fetsch et al. 2007; Vallar et al. 1999). Since some of these coordinate systems seem to use in the prefrontal cortex to encode spatial information of stimuli, additional studies are need to understand what coordinate system is used to encode spatial information in the prefrontal cortex under a variety of conditions.

\subsection{Interactions between spatial and non-spatial working memory in the prefrontal cortex}

Delay-period activity has been observed in many prefrontal neurons while monkeys performed spatial as well as non-spatial working memory tasks. Delay-period activity represents a particular type of spatial information (e.g., the spatial position of the visual cue) as well as a particular feature of the visual object. These observations raise two questions; does each prefrontal neuron encode either spatial information or object information?, and is the spatial distribution of prefrontal neurons that encode spatial information different from that of neurons that encode object information?

Wilson et al. (1993) examined single-neuron activity in the prefrontal cortex using the ODR task and an object discrimination task. They found neurons that responded selectively to objects and faces in the ventral convexity of the prefrontal cortex. Since neurons in the cortex surrounding the principal sulcus showed delay-period activity while monkeys performed the ODR task (Funahashi et al. 1989, 1993b), they concluded that the prefrontal cortex contains separate information-processing domains: one for processing spatial information (where an 
object was) and another for processing non-spatial information (what the object was).

However, Rao et al. (1997) examined prefrontal single-neuron activity while monkeys performed a delay task that required working memory of both object (what) and location (where), and found that, although some neurons had either object-tuned (what) or location-tuned (where) delay-period activity, about half (52\%) of prefrontal neurons had both object- and location-tuned delay-period activity. This result indicates that spatial (where) and non-spatial (what) information are integrated in individual prefrontal neurons. Rainer et al. (1998a) further examined how prefrontal neurons represent conjunctions of "what" and "where" information. They examined memory fields of prefrontal neurons by presenting visual objects at various positions within the visual field and forcing monkeys to remember both the object itself and the position where the object was presented. They found that the activities of many prefrontal neurons represented object information. At the same time, they found that these neurons had memory fields for spatial information of the object and that these memory fields were located mainly in the contralateral visual field. Based on these results, they suggested that prefrontal neurons play a role in constructing a unified representation of a visual scene by simultaneously representing both spatial and object information.

Hoshi et al. (2000) examined prefrontal activity using a location-matching task and a shape-matching task, and found that a majority (54\%) of prefrontal neurons represented spatial information, while neurons that represented only non-spatial information or neurons that represented both spatial and non-spatial information were in the minority. In addition, neurons that represented spatial information and neurons that represented non-spatial information were intermingled within the prefrontal area, although more neurons that represented spatial information were found in the posterior part of the lateral prefrontal cortex while more neurons fthat represented non-spatial information were found in the anterior part.

These results indicate that, although the delay-period activity observed in most 
prefrontal neurons represents spatial information, a substantial number of prefrontal neurons encode both spatial and non-spatial information and play important roles in integrating both types of information. In addition, prefrontal neurons exhibiting delay-period activity are usually widely distributed within the prefrontal cortex. The spatial distribution of neurons exhibiting delay-period activity within the prefrontal cortex overlaps that of neurons exhibiting other task-related activities (Carlson et al. 1997). The overlapping of the spatial distributions of neurons representing different modalities of information within the prefrontal cortex could play important roles in the integration and processing of multimodal information.

\section{Information processing from visuospatial representation to motor representation}

In the delayed-response task, the subject is instructed regarding what behavior needs to be performed during the response period through the use of visuospatial cues. Based on the instruction provided during the cue period, the subject needs to perform a particular behavioral response. Visuospatial information must be transformed into motor information. Therefore, the transformation of visuospatial information into motor information in the delayed-response task could be an appropriate model for examining the neural mechanisms of information processing in the brain.

When we consider the mechanism of information processing in the brain, this process can be depicted as a change in the information that is represented by the activity of a population of neurons or a neural network. Furthermore, this change in information can be understood to be the result of dynamic interactions among neurons in the network. Several studies have shown that the information represented by prefrontal activity changes as the task trial progresses. For example, using a delayed paired association task, Rainer et al. (1999) showed that, although prefrontal activity primarily 
represented characteristics of the sample stimuli (sensory-related coding) during the early phase of the delay period, this activity began to represent characteristics of anticipated targets (prospective coding) toward the end of the delay period. Similarly, using a spatial delayed matching-to-sample task, Sawaguchi and Yamane (1999) showed that spatial information was tuned broadly by delay-period activity in the early phase of the delay period. However, the proportion of neurons that exhibited sharper spatial tuning and high spatial discriminability increased in the later phase of the delay period. Further, Asaad et al. (1998) showed that neural activity conveyed the direction of impending eye movement progressively earlier in successive trials while monkeys performed arbitrary cue-response association tasks. Quintana and Fuster (1999) observed prefrontal neurons that were attuned to the color of the visual cue and neurons that were attuned to the response direction while monkeys performed working memory tasks using color cues. They also found that the discharge of neurons that were attuned to the cue color gradually diminished during the delay period, whereas the discharge of neurons that were attuned to response directions gradually increased. These results indicate that the temporal pattern of neural discharge changes as the trial progresses depending on the requirements of the task. Thus, these results suggest that the modulation of the temporal pattern of neural discharges reflects the change in the information represented by the neuron and that the information represented by the temporal pattern of the firing of a neuron or a population of neurons gradually changes as the trial progresses.

Takeda and Funahashi (2002) examined dorsolateral prefrontal activity using two kinds of oculomotor delayed-response tasks (ODR and R-ODR tasks). In the ODR task, the monkey was required to make a saccade to the direction where the visual cue was presented, whereas in the R-ODR task, the monkey was required to make a saccade $90^{\circ}$ clockwise from the direction where the visual cue was presented. They compared the directional selectivity of delay-period activity between these two task conditions in 
many prefrontal neurons and showed that the majority of delay-period activity represented the direction of the visual cue (representing retrospective information), whereas the minority of delay-period activity and the majority of response-period activity represented the direction of the saccade (representing prospective information). The evidence that the prefrontal cortex includes neurons that represent either retrospective or prospective information suggests that the prefrontal cortex includes a neural mechanism for transforming visuospatial information into motor information. Since the characteristics of both visuospatial and motor information can be depicted as a difference in the directional preference observed in neural activity, the transformation of visuospatial information into motor information can be demonstrated as a temporal change in the directional preference observed in a population of neural activities as the task progresses.

Takeda and Funahashi (2004) used population vectors to demonstrate the temporal change in the directional preference observed in a population of prefrontal activities as a trial progressed in both the ODR and R-ODR tasks. Figure 9A shows temporal changes in population vectors calculated from a population of dorsolateral prefrontal activities in the $180^{\circ}$ trial of the ODR task. Population vectors were mostly directed toward the $180^{\circ}$ direction. Since the direction of the visual cue and the direction of the saccade were the same in the ODR task, this result indicates that directional information is maintained by a population of prefrontal neurons during the delay period (see Fig. 9B). Figure 9C shows a temporal change in population vectors calculated from a population of dorsolateral prefrontal activities in the $180^{\circ}$ trial of the R-ODR task. In this trial, the visual cue was presented at the $180^{\circ}$ direction and the direction of the correct saccade was in the $90^{\circ}$ direction. In this condition, population vectors were directed toward the $180^{\circ}$ direction at the beginning of the delay period. However, population vectors began to rotate toward the $90^{\circ}$ direction in the middle of the delay period, continued to rotate slowly from the $180^{\circ}$ direction to the $90^{\circ}$ direction during the 
late half of the delay period, and finally were directed toward the $90^{\circ}$ direction in the response period (see Fig. 9D). This result indicates that the information represented by a population of dorsolateral prefrontal activities changes during the delay period, in that, although a population of prefrontal neurons initially hold information regarding the direction of the visual cue, as the trial progresses, this information gradually changes to information regarding the motor response. Thus, these results indicate that information processing in the brain can be depicted as the temporal change in the information represented by a population of neurons. In the delayed-response task, visuospatial information needs to be transformed into motor information, since the visual stimulus is used as a cue and since the subject is required to perform a motor behavior as a response to get a reward. Therefore, the visuospatial information provided as the cue should be transformed into motor information. These studies indicate that prefrontal neurons participate in this transformation process, and that this transformation process can be visualized using an appropriate method, such as a population vector analysis.

Fuster (2008) proposed that the mediation of cross-temporal contingency is an important function of the prefrontal cortex. He stated that the delay period is when the cross-temporal bridging of sensory-to-motor information transformation occurs and that this is a dynamic process for the internal transformation of information as well as a process of cross-temporal matching. Experimental results support the notion that the prefrontal cortex plays a significant role in mediating the cross-temporal contingency. These results also suggest that both retrospective-encoding and prospective-encoding delay-period activities observed in prefrontal neurons contribute significantly to the dynamic process related to the internal transformation of information.

\section{Interactions among prefrontal neurons in spatial information processing}




\subsection{Information flow within the prefrontal cortex}

While monkeys performed the ODR task, only cue- (C), only delay- (D), or only response-period $(\mathrm{R})$ activity, or a combination of these activities $(\mathrm{CD}, \mathrm{CR}, \mathrm{DR}$, or CDR) was observed as task-related activity in each prefrontal neuron. Funahashi and Inoue (2000) examined the interactions of prefrontal neurons by a cross-correlation analysis in which they simultaneously isolated two single-neuron activities and demonstrated the presence of information flow from neurons with cue-period activity to neurons with oculomotor activity through neurons with delay-period activity in the dorsolateral prefrontal cortex. In addition, the information that was represented by each task-related activity can be identified by comparing the spatial tuning of this activity between the ODR and R-ODR tasks (Takeda and Funahashi 2002). Takeda and Funahashi (2007) characterized each prefrontal neuron based on which task-related activity was exhibited and what information (either cue direction or saccade direction) the task-related activity represented. Using this method, they classified prefrontal neurons into 9 groups (Ccue, Dcue, Dsac, CcueDcue, DcueRcue, DsacRsac, DcueRsac, CcueDcueRcue and CcueDcueRsac) (Fig. 10). In this classification, task-related activity that represented information about the cue direction was indicated by 'cue,' while task-related activity that represented information of the saccade direction was indicated by 'sac.' For example, neurons that were classified as CcueDcueRsac had cue- and delay-period activities that represented the cue direction and response-period activity that represented the saccade direction.

They found that the preferred directions were similar between cue- and delay-period activities in the CcueDcue, CcueDcueRcue, and CcueDcueRsac groups. Since these task-related activities were observed in the same neurons, the directional selectivity of delay-period activity could be affected by the directional selectivity of cue-period activity. The preferred directions were also similar between the delay- and response-period activities in the DcueRcue, CcueDcueRcue, and DsacRsac groups, 
which suggests that the directional selectivity of delay-period activity could affect the directional selectivity of response-period activity in these neurons. On the other hand, based on a comparison of the temporal profile of delay-period activity, the directional selectivity of cue-period activity in the Ccue, CcueDcue, and CcueDcueRcue groups could contribute to the directional selectivity of delay-period activity in the CcueDcue, CcueDcueRcue, Dcue, and DcueRcue groups. In addition, the directional selectivity of saccade-related activity in the DsacRsac group could be affected by the directional selectivity of delay-period activity in the Dsac and DsacRsac groups.

Thus, while monkeys performed the ODR task, information flow from neurons with cue-period activity to those with oculomotor activity through neurons with delay-period activity is present in the prefrontal cortex (Fig. 11). Visuospatial inputs from the posterior cortices first activate prefrontal neurons that exhibit only cue-period activity (Ccue). This activation would be transferred to prefrontal neurons with both cue- and delay-period activities (СсиеDcue) and then transferred to prefrontal neurons with only delay-period activity (Dcue). Since these prefrontal neurons receive visuospatial inputs, both cue- and delay-period activities observed in these neurons represent visuospatial information. However, during the delay period, prospective motor information must be generated and this information is maintained in prefrontal neurons that exhibit delay-period activity (Dsac). This information would be transferred to prefrontal neurons with both delay- and response-period activities (DsacRsac) and then transferred to prefrontal neurons with only response-period activity (Rsac).

\subsection{How is prospective motor information generated in the prefrontal cortex?}

A comparison of the directional selectivity of delay-period activity between the ODR and R-ODR tasks revealed that delay-period activity that encodes either retrospective visuospatial information or prospective saccade information is present in the prefrontal cortex (Takeda and Funahashi 2002). However, delay-period activity that 
encodes both visual and saccade information simultaneously may not be present in the prefrontal cortex. Therefore, prospective motor information needs to be generated in the prefrontal cortex by some method. One possibility is that some brain structure provides motor information to the prefrontal cortex, and the mediodorsal nucleus of the thalamus (MD) is a potential candidate (Watanabe and Funahashi 2012). This possibility is supported by several observations. For example, Isseroff et al. (1982) showed that spatial memory was impaired in monkeys by damage to the MD. Watanabe and Funahashi (2004a) showed that same task-related activities were observed in both the MD and the prefrontal cortex while monkeys performed the ODR task and that a majority of saccade-related activity in the MD was pre-saccadic. In addition, Watanabe and Funahashi (2004b) showed that more neurons with delay-period activity encoded prospective motor information in the MD while few neurons with delay-period activity encoded motor information in the dorsolateral prefrontal cortex. It is well known that the MD has strong reciprocal connections with the prefrontal cortex. Thus, the MD must be a candidate brain structure that provides information regarding prospective motor information to the prefrontal cortex (Fig. 11).

\subsection{Functional interactions among prefrontal neurons}

The dynamic and flexible changes in functional interaction among prefrontal neurons are likely to be key factors for understanding the neuronal mechanisms of information processing. In the prefrontal cortex, Wilson et al. (1994) showed that pyramidal and non-pyramidal neurons often showed different types of responses (e.g., pyramidal neurons exhibited an excitatory response while non-pyramidal neurons exhibited an inhibitory response) and that the timing of excitatory and inhibitory responses appeared to be phased. This result suggests the presence of functional interactions between pyramidal and non-pyramidal neurons. Rao et al. (1999) used a cross-correlation analysis to examine functional interactions between pyramidal and 
non-pyramidal neurons in the prefrontal cortex, and found inhibitory interactions between pyramidal neurons and adjacent non-pyramidal interneurons. Further, Rao et al. (2000) showed that GABAergic interneurons played an important role in the development of spatial tuning in the prefrontal cortex. Funahashi and Inoue (2000) and Constantinidis et al. (2001b) applied a cross-correlation analysis to simultaneously isolated pairs of prefrontal activities during ODR performance and examined functional interactions between task-related prefrontal neurons. When both neurons of the examined pairs exhibited delay-period activity, these neurons tended to show a similar directional preference and excitatory interactions. Interactions between task-related neurons with different directional preferences increased as the trial progressed. Excitatory interactions among neurons with delay-period activity were frequently observed in the prefrontal cortex. For each neuron, the magnitude of activity changes depending on the trial condition, the temporal context of the trial, and trial events. Therefore, the strength of functional interactions could change depending on the trial conditions or the temporal context of the trial. In fact, the height of the peak of the cross-correlogram changed depending on the cue conditions. The strength of the interaction changes dynamically depending on the condition of the trial and this dynamic change may contribute to stabilize the information represented on the prefrontal neural network (Renart et al. 2003). Thus, dynamic and flexible changes in functional interactions among neurons with delay-period activity could be an important element for understanding the neural mechanisms of information processing.

\section{Conclusions}

Spatial representation and its functions in the prefrontal cortex have been examined using a variety of behavioral tasks. Since Jacobsen (1936) first reported that bilateral lesions of the lateral prefrontal cortex caused an impairment of 
delayed-response performance in monkeys, the delayed-response task has been widely used to examine spatial functions of the prefrontal cortex. In this task, spatial information regarding the baiting position must be temporarily maintained if the subject is to make a correct response and obtain a reward. Therefore, the delayed-response task is appropriate for examining the mechanism of spatial representation in the brain. In addition, the concept of working memory to explain prefrontal functions, which was first proposed by Goldman-Rakic (1987), has greatly facilitated our understanding of the nature and functions of spatial representation in the prefrontal cortex. While single-neuron recording studies have revealed some aspects of spatial representation in the prefrontal cortex by showing characteristics of visual as well as auditory receptive fields, the detailed analysis of directional delay-period activity in spatial working memory tasks has provided copious important information regarding spatial representation in the prefrontal cortex. With the use of oculomotor versions of the delayed-response task (e.g., ODR task), it has been shown that directional delay-period activity is a neural correlate of the mechanism for temporarily maintaining information and that a great majority of this activity represents spatial information regarding the visual cue. The spatial tuning of delay-period activity differs from neuron to neuron. Therefore, each prefrontal neuron represents information presented in a different area of the visual space (e.g., memory field), although there is extensive overlap among the memory fields represented by each of these neurons.

In addition, many prefrontal neurons respond to visual or auditory stimuli and have visual or auditory receptive fields, respectively. Further, many prefrontal neurons exhibit saccade-related activity and most saccade-related activities are directionally selective. Thus, many prefrontal neurons that participate in sensory information processing, motor information processing, and cognitive information processing exhibit spatial selectivity. Therefore, these neurons are important components for the operation of spatial information processing in the prefrontal cortex. Cross-correlation analyses of 
simultaneously isolated single-neuron activities have revealed information flow from sensory-related neurons to motor-related neurons through memory-related neurons in the prefrontal cortex during delayed-response performance. Population vector analyses of directionally selective task-related activities have made it possible to visualize the change in the spatial information represented by a population of prefrontal neurons during delayed-response performance. Information flow from sensory-related neurons to motor-related neurons and the change in spatial representation as the trial progresses both depend on dynamic interactions among neurons that exhibit different task-related activities and represent different information. Although our understanding of these subjects is gradually increasing (e.g., Constantinidis et al. 2001b; Funahashi and Inoue 2000; Takeda and Funahashi 2004), further studies are needed to elucidate the nature and characteristics of functional interactions among prefrontal neurons. In addition, functional interactions among neurons can be dynamically modulated by the effects of neurotransmitters. The dynamic modulation of prefrontal single-neuron activity by the application of neurochemicals (e.g., dopamine and dopamine-related neurochemicals) has been demonstrated by Vijayraghavan et al. (2007), Wang et al. (2004), and Williams and Goldman-Rakic (1995). Further studies are needed on the effects of a variety of neurotransmitters on the dynamic modulation of neural activity as well as on the interactions among neurons during spatial working memory performance. We also need to consider the functional significance of such modulation on spatial information processes.

As shown in Fig. 11, we suggested that information provided from the MD is necessary to construct spatial information for motor performance in the prefrontal cortex. However, information provided from other cortical areas might be necessary to construct directional information for saccades. Therefore, we also need to understand the nature and the characteristics of interactions between the prefrontal cortex and other cortical and subcortical areas. Although directionally selective activity related to the 
behavioral response is observed in the prefrontal cortex, this activity is not directly related to the movement of the hand or the arm itself, but rather is related to the movement of the cursor on the computer monitor (Mushiake et al. 2006). In addition, although a response to the visual cue can be observed in many prefrontal neurons, this response is not directly related to the physical properties of the visual cue, but rather is related to the meaning of the visual cue, such as whether it is a Go or No-Go response (e.g., Watanabe 1986a, b). The mechanisms that underlie how these activities are constructed in the brain and how the prefrontal cortex participates in these mechanisms need to be elucidated. Space representation in the prefrontal cortex may play an important role in these studies. 


\section{Acknowledgment}

This work was supported by Grants-in-Aid for Scientific Research from the Japanese Ministry of Education, Culture, Sports, Science, and Technology (MEXT) (18020016, 21240024, 23135518). This work was also supported by the Global COE Program to Kyoto University (D07) from the Japanese Ministry of Education, Culture, Sports, Science, and Technology. 


\section{References}

Andersen, R.A., Asanuma, C., Cowan, W.M., 1985a. Callosal and prefrontal associational projecting cell populations in area 7A of the macaque monkey: a study using retrogradely transported fluorescent dyes. J. Comp. Neurol. 232, 443-455.

Andersen, R.A., Essick, G.K., Siegel, R.M., 1985b. Encoding of spatial location by posterior parietal neurons. Science 230, 456-458.

Andersen, R.A., Mountcastle, V.B., 1983. The influence of the angle of gaze upon the excitability of the light-sensitive neurons of the posterior parietal cortex. J. Neurosci. $3,532-548$.

Arcaro, M.J., McMains, S.A., Singer, B.D., Kastner, S., 2009. Retinotopic organization of human ventral visual cortex. J. Neurosci., 29, 10638-10652.

Arcaro, M.J., Pinsk, M.A., Li, X., Kastner, S., 2011. Visuotopic organization of macaque posterior parietal cortex: a functional magnetic resonance imaging study. J. Neurosci. 31, 2064-2078.

Asaad, W.F., Rainer, G., Miller, E.K., 1998. Neural activity in the primate prefrontal cortex during associative learning. Neuron 21, 1399-1407.

Averbeck, B.B., Romanski, L.M., 2006. Probabilistic encoding of vocalizations in macaque ventral lateral prefrontal cortex. J. Neurosci. 26, 11023-11033.

Azuma, M., Suzuki, H., 1984. Properties and distribution of auditory neurons in the dorsolateral prefrontal cortex of the alert monkey. Brain Res. 298, 343-346.

Baker, S.C., Frith, C.D., Frackowiak, R.S.J., Dolan, R.J., 1996. Active representation of shape and spatial location in man. Cereb. Cortex 6, 612-619.

Barone, P., Joseph, J.-P., 1989. Prefrontal cortex and spatial sequencing in macaque monkey. Exp. Brain Res. 78, 447-464.

Beurze, S.M., Roni, I., Pisella, L., Mendendorp, W.P., 2010. Reference frames for reach planning in human parietofrontal cortex. J. Neurophysiol. 104, 1736-1745. 
Boch, R.A., Goldberg, M.E., 1989. Participation of prefrontal neurons in the preparation of visually guided eye movements in the rhesus monkey. J. Neurophysiol. 61, 1064-1084.

Boussaoud, D., 1995. Primate premotor cortex: modulation of preparatory neuronal activity by gaze angle. J. Neurophysiol. 73, 886-890.

Boussaoud, D., Jouffrais, C., Bremmer, F., 1998. Eye position effects on the neuronal activity of dorsal premotor cortex in the macaque monkey. J. Neurophysiol. 80, 1132-1150.

Brown, M.R.G., DeSouza, J.F.X., Goltz, H.C., Ford, K., Menon, R.S., Goodale, M.A., Everling, S., 2004. Comparison of memory- and visually guided saccades using event-related fMRI. J. Neurophysiol. 91, 873-889.

Bruce, C.J., Goldberg, M.E., 1985. Primate frontal eye fields. I. Single neurons discharging before saccades. J. Neurophysiol. 53, 603-635.

Buschman, T.J., Miller, E.K., 2007. Top-down versus bottom-up control of attention in the prefrontal and posterior parietal cortices. Science 315, 1860-1862.

Bushara, K.O., Weeks, R.A., Ishii, K., Catalan, M.-J., Tian, B., Rauschecker, J.P., Hallett, M., 1999. Modality-specific frontal and parietal areas for auditory and visual spatial localization in humans. Nature Neurosci. 2, 759-766.

Butters, N., Pandya, D., Sanders, K., Dye, P., 1971. Behavioral deficits in monkeys after selective lesions within the middle third of sulcus principalis. J. Comp. Physiol. Psychol. 76, 8-14.

Carlson, S., Rama, P., Tanila, H., Linnankoski, I., Mansikka, H., 1997. Dissociation of mnemonic coding and other functional neuronal processing in the monkey prefrontal cortex. J. Neurophysiol. 77, 761-774.

Carrozzo, M., McIntyre, J., Zago, M., Lacquaniti, F., 1999. Viewer-centered and body-centered frames of reference in direct visuomotor transformation. Exp. Brain Res. 129, 201-210. 
Cavada, C., Goldman-Rakic, P.S., 1989. Posterior parietal cortex in rhesus monkey. II. Evidence for segregated corticocortical networks linking sensory and limbic areas with the frontal lobe. J. Comp. Neurol. 287, 422-445.

Chafee, M.V., Goldman-Rakic, P.S., 1998. Matching patterns of activity in primate prefrontal area $8 \mathrm{a}$ and parietal area 7ip neurons during a spatial working memory. J. Neurophysiol. 79, 2919-2940.

Ciaramelli, E., 2008. The role of ventromedial prefrontal cortex in navigation: a case of impaired wayfinding and rehabilitation. Neuropsychologia 46, 2099-2105.

Cohen, Y.E., Russ, B.E., Gifford III, G.W., Kiringoda, R., MacLean, K.A., 2004. Selectivity for the spatial and nonspatial attributes of auditory stimuli in the ventrolateral prefrontal cortex. J. Neurosci. 24, 11307-11316.

Constantinidis, C., Franowicz, M.N., Goldman-Rakic, P.S., 2001a. The sensory nature of mnemonic representation in the primate prefrontal cortex. Nature Neurosci. 4, 311-316.

Constantinidis, C., Franowicz, M.N., Goldman-Rakic, P.S., 2001b. Coding specificity in cortical microcircuits: a multiple-electrode analysis of primate prefrontal cortex. J. Neurosci. 21, 3646-3655.

Courtney, S.M., Petit, L., Maisog, J.M., Ungerleider, L.G., Haxby, J.V., 1998. An area specialized for spatial working memory in human frontal cortex. Science 279, 1347-1351.

Courtney, S.M., Ungerleider, L.G., Keil, K., Haxby, J.V., 1996. Object and spatial visual working memory activate separate neural systems in human cortex. Cereb. Cortex 6, 39-49.

Curtis, C.E., D’Esposito, M., 2003. Persistent activity in the prefrontal cortex during working memory. Trends Cog. Sci. 7, 415-423.

Curtis, C.E., D'Esposito, M., 2006. Selection and maintenance of saccade goals in the human frontal eye fields. J. Neurophysiol. 95, 3923-3927. 
Curtis, C.E., Rao, V.Y., D’Esposito, M., 2004. Maintenance of spatial and motor codes during oculomotor delayed response tasks. J. Neurosci. 24, 3944-3952.

Desimone, R., Albright, T.D., Gross, C.G., Bruce, C., 1984. Stimulus-selective properties of inferior temporal neurons in the macaque. J. Neurosci. 4, 2051-2062.

D’Esposito, M., Aguirre, G.K., Zarahn, E., Ballard, D., Shin, R.K., Lease, J., 1998. Functional MRI studies of spatial and nonspatial working memory. Cognit. Brain Res. 7, 1-13.

D’Esposito, M., Ballard, D., Zarahn, E., Aguirre, G.K., 2000. The role of prefrontal cortex in sensory memory and motor preparation: an event-related fMRI study. Neuroimage 11, 400-408.

di Pellegrino, G., Wise, S.P., 1993. Visuospatial versus visuomotor activity in the premotor and prefrontal cortex of a primate. J. Neurosci. 13, 1227-1243.

Engel, S.A., Glover, G.H., Wandell, B.A., 1997. Retinotopic organization in human visual cortex and the spatial precision of functional MRI. Cereb. Cortex 7, 181-192.

Fetsch, C.R., Wang, S., Gu, Y., DeAngelis, G.C., Angelaki, D.E., 2007. Spatial reference frames of visual, vestibular, and multimodal heading signals in the dorsal subdivision of the medial superior temporal area. J. Neurosci. 27, 700-712.

Fogassi, L., Gallese, V., Fadiga, L., Luppino, G., Matelli, M., Rizzolatti, G., 1996. Coding of peripersonal space in inferior premotor cortex (area F4). J. Neurophysiol. $76,141-157$.

Freedman, M., Oscar-Berman, M., 1986. Bilateral frontal lobe disease and selective delayed response deficits in humans. Behav. Neurosci. 100, 337-342.

Fukushima, T., Hasegawa, I., Miyashita, Y., 2004. Prefrontal neuronal activity encodes spatial target representations sequentially updated after nonspatial target-shift cues. J. Neurophysiol. 91, 1367-1380.

Funahashi, S., 1983. Responses of monkey prefrontal neurons during a visual tracking task reinforced by substantia innominata self-stimulation. Brain Res. 276, 267-276. 
Funahashi, S., 2001. Neuronal mechanisms of executive control by the prefrontal cortex. Neurosci. Res. 39, 147-165.

Funahashi, S., Kubota, K., 1994. Working memory and prefrontal cortex. Neurosci. Res. $21,1-11$.

Funahashi, S., Bruce, C.J., Goldman-Rakic, P.S., 1989. Mnemonic coding of visual space in the monkey's dorsolateral prefrontal cortex. J. Neurophysiol. 61, 331-349.

Funahashi, S., Bruce, C.J., Goldman-Rakic, P.S., 1990. Visuospatial coding in primate prefrontal neurons revealed by oculomotor paradigms. J. Neurophysiol. 63, 814-831.

Funahashi, S., Bruce, C.J., Goldman-Rakic, P.S., 1991. Neuronal activity related to saccadic eye movements in the monkey's dorsolateral prefrontal cortex. J. Neurophysiol. 65, 1464-1483.

Funahashi, S., Bruce, C.J., Goldman-Rakic, P.S., 1993a. Dorsolateral prefrontal lesions and oculomotor delayed-response performance: evidence for mnemonic "scotomas." J. Neurosci. 13, 1479-1497.

Funahashi, S., Chafee, M.V., Goldman-Rakic, P.S., 1993b. Prefrontal neuronal activity in rhesus monkeys performing a delayed anti-saccade task. Nature 365, 753-756.

Funahashi, S., Inoue, M., 2000. Neuronal interactions related to working memory processes in the primate prefrontal cortex revealed by cross-correlation analysis. Cereb. Cortex 10, 535-551.

Funahashi, S., Inoue, M., Kubota, K., 1993c. Delay-related activity in the primate prefrontal cortex during sequential reaching tasks with delay. Neurosci. Res. 18, $171-175$.

Funahashi, S., Inoue, M., Kubota, K., 1997. Delay-period activity in the primate prefrontal cortex encoding multiple spatial positions and their order of presentation. Behav. Brain Res. 84, 203-223. 
Funahashi, S., Takeda, K., 2002. Information processes in the primate prefrontal cortex in relation to working memory processes. Rev. Neurosci. 13, 313-345.

Funahashi, S., Takeda, K., Watanabe, Y., 2004. Neural mechanisms of spatial working memory: contributions of the dorsolateral prefrontal cortex and the thalamic mediodorsal nucleus. Cogn. Affect. Behav. Neurosci. 4, 409-420.

Fuster, J.M., 1973. Unit activity in prefrontal cortex during delayed-response performance: neuronal correlates of transient memory. J. Neurophysiol. 36, 61-78.

Fuster, J.M., 2008. The Prefrontal Cortex, Fourth edition. Academic Press, New York.

Fuster, J.M,, Alexander, G.E., 1971. Neuron activity related to short-term memory. Science 173, 652-654.

Fuster, J.M., Bauer, R.H., Jervey, J.P., 1982. Cellular discharge in the dorsolateral prefrontal cortex of the monkey in cognitive tasks. Exp. Neurol. 77, 679-694.

Galletti, C., Battaglini, P., 1989. Gaze-dependent visual neurons in area V3A of monkey prestriate cortex. J. Neurosci. 9, 1112-1125.

Galletti, C., Battaglini, P.P., Fattori, P., 1993. Parietal neurons encoding spatial locations in craniotopic coordinates. Exp. Brain Res. 96, 221-229.

Gardner, J.L., Merriam, E.P., Movshon, J.A., Heeger, D.J., 2008. Maps of visual space in human occipital cortex are retinotopic, not spatiotopic. J. Neurosci. 28, 3988-3999.

Genovesio, A., Brasted, P.J., Wise, S.P., 2006. Representation of future and previous spatial goals by separate neural populations in prefrontal cortex. J. Neurosci. 26, $7305-7316$.

Genovesio, A., Tsujimoto, S., Wise, S.P., 2011. Prefrontal cortex activity during the discrimination of relative distance. J. Neurosci. 31, 3968-3980.

Glahn, D.C., Kim, J., Cohen, M.S., Poutanen, V.P., Therman, S., Bava, S., van Erp, T.G.M., Manninen, M., Huttunen, M., Lonnqvist, J., Standertskjold-Nordenstam, C.G., Cannon, T.D., 2002. Maintenance and manipulation in spatial working 
memory: dissociations in the prefrontal cortex. Neuroimage 17, 201-213.

Goldman-Rakic P.S., 1987. Circuitry of primate prefrontal cortex and regulation of behavior by representational memory. In: Plum, F. (Ed.), Handbook of Physiology, Section 1, The Nervous System, Vol. V, Higher Functions of the Brain. American Physiological Society, Bethesda, MD, pp. 373-417.

Goldman-Rakic, P.S., 1996a. Regional and cellular fractionation of working memory. Proc. Natl. Acad. Sci. USA 93, 13473-13480.

Goldman-Rakic, P.S., 1996b. The prefrontal landscape: implications of functional architecture for understanding human mentation and the central executive. Phil. Trans. R. Soc. Lond. B 351, 1445-1453.

Goldman-Rakic, P.S., 1998. The prefrontal landscape: implications of functional architecture for understanding human mentation and the central executive. In: Roberts, A.C., Robbins, T.W., Weiskrantz, L. (Eds.), The Prefrontal Cortex: Executive and Cognitive Functions. Oxford University Press, Oxford, UK, pp. 87-102.

Goldman-Rakic, P.S., Funahashi, S., Bruce, C.J., 1990. Neocortical memory circuits. Cold Spring Harbor Symp. Quant. Biol. 55, 1025-1038.

Gottlieb, J.P., Kusunoki, M., Goldberg, M.E., 1998. The representation of visual salience in monkey parietal cortex. Nature 391, 481-484.

Graziano, M.S.A., Hu, X.T., Gross, C.G., 1997. Visuospatial properties of ventral premotor cortex. J. Neurophysiol. 77, 2268-2292.

Gross, C.G., Rocha-Miranda, C.E., Bender, D.B., 1972. Visual properties of neurons in inferotemporal cortex of the macaque. J. Neurophysiol. 35, 96-111.

Hagler Jr., D.J., Sereno, M.I., 2006. Spatial maps in frontal and prefrontal cortex. Neuroimage 29, 567-577.

Hasegawa, R., Matsumoto, M., Mikami, A., 2000. Search target selection in monkey prefrontal cortex. J. Neurophysiol. 84, 1692-1696. 
Hikosaka, O., Wurtz, R.H., 1983. Visual and oculomotor functions of monkey substantia nigra pars reticulata. III. Memory-contingent visual and saccade responses. J. Neurophysiol. 49, 1268-1284.

Horst, N.K., Laubach, M., 2009. The role of rat dorsomedial prefrontal cortex in spatial working memory. Neuroscience 165, 444-456.

Hoshi, E., Tanji, J., 2004. Area-selective neuronal activity in the dorsolateral prefrontal cortex for information retrieval and action planning. J. Neurophysiol. 91, 2707-2722.

Hoshi, E., Shima, K., Tanji, J., 1998. Task-dependent selectivity of movement-related neuronal activity in the primate prefrontal cortex. J. Neurophysiol. 80, 3392-2297.

Hoshi, E., Shima, K., Tanji, J., 2000. Neuronal activity in the primate prefrontal cortex in the process of motor selection based on two behavioral rules. J. Neurophysiol. 83, 2355-2373.

Ikkai, A., Curtis, C.E., 2011. Common neural mechanisms supporting spatial working memory, attention and motor intention. Neuropsychologia 49, 1428-1434.

Inoue, M., Funahashi, S., 2002. Prefrontal delay-period activity is affected by visual cues presented outside the memory field. Neuroreport 13, 2097-2101.

Isseroff, A., Rosvold, H.E., Galkin, T.W., Goldman-Rakic, P.S., 1982. Spatial memory impairments following damage to the mediodorsal nucleus of the thalamus in rhesus monkeys. Brain Res. 232, 97-113.

Ito, S., 1982. Prefrontal unit activity of macaque monkey during auditory and visual reaction time tasks. Brain Res. 247, 39-47.

Izawa, Y., Suzuki, H., Shinoda, Y., 2004. Suppression of visually and memory-guided saccades induced by electrical stimulation of the monkey frontal eye field. I. Suppression of ipsilateral saccades. J. Neurophysiol. 92, 2248-2260.

Jacobsen, C.F., 1936. Studies of cerebral function in primates. I. The functions of the frontal association areas in monkeys. Comp. Psychol. Monog. 13, 1-60.

Jenkins, I.H., Brooks, D.J., Nixon, P.D., Frackowiak, R.S.J., Passingham, R.E., 1994. 
Motor sequence learning: a study with positron emission tomography. J. Neurosci. $14,3775-3790$.

Jonides, J., Smith, E.E., Koeppe, R.A., Awh, E., Minoshima, S., Mintun, M.A., 1993. Spatial working memory in humans as revealed by PET. Nature 363, 623-625.

Joseph, J.P., Barone, P., 1987. Prefrontal unit activity during a delayed oculomotor task in the monkey. Exp. Brain Res. 67, 460-468.

Kastner, S., DeSimone, K., Konen, C.S., Szczepanski, S.M., Weiner, K.S., Schneider, K.A., 2007. Topographic maps in human frontal cortex revealed in memory-guided saccade and spatial working-memory tasks. J. Neurophysiol. 97, 3494-3507.

Kawamura, K., Naito, J., 1984. Corticocortical projections to the prefrontal cortex in the rhesus monkey investigated with horseradish peroxidase techniques. Neurosci. Res. 1, 89-103.

Kikuchi-Yorioka, Y., Sawaguchi, T., 2000. Parallel visuospatial and audiospatial working memory processes in the monkey dorsolateral prefrontal cortex. Nature Neurosci. 3, 1075-1076.

Kojima, S., Goldman-Rakic, P.S., 1982. Delay-related activity of prefrontal neurons in rhesus monkeys performing delayed response. Brain Res. 248, 43-49.

Kojima, S., Goldman-Rakic, P.S., 1984. Functional analysis of spatially discriminative neurons in prefrontal cortex of rhesus monkey. Brain Res. 291, 229-240.

Kojima, S., Matsumura, M., Kubota, K., 1981. Prefrontal neuron activity during delayed-response performance without imperative GO signals in the monkey. Exp. Neurol. 74, 396-407.

Kojima, Y., Soetedjo, R., Fuchs, A.F., 2010. Behavior of the oculomotor vermis for five different types of saccade. J. Neurophysiol. 104, 3667-3676.

Kolster, H., Peeters, R., Orban, G.A., 2010. The retinotopic organization of the human middle temporal area MT/V5 and its cortical neighbors. J. Neurosci., 30, 9801-9820.

Kubota, K., Funahashi, S., 1982. Direction-specific activities of dorsolateral prefrontal 
and motor cortex pyramidal tract neurons during visual tracking. J. Neurophysiol. 47, $362-376$.

Kubota, K., Iwamoto, T., Suzuki, H., 1974. Visuokinetic activities of primate prefrontal neurons during delayed-response performance. J. Neurophysiol. 37, 1197-1212.

Kubota, K., Komatsu, H., 1985. Neuron activities of monkey prefrontal cortex during the learning of visual discrimination tasks with go/no-go performances. Neurosci. Res. 3, 106-129.

Kubota, K., Niki, H., 1971. Prefrontal cortical unit activity and delayed alternation performance in monkeys. J. Neurophysiol. 34, 337-347.

Kubota, K., Tonoike, M., Mikami, A., 1980. Neuronal activity in the monkey dorsolateral prefrontal cortex during a discrimination task with delay. Brain Res. 183, $29-42$.

Kurata, K., 2007. Laterality of movement-related activity reflects transformation of coordinates in ventral premotor cortex and primary motor cortex of monkeys. J. Neurophysiol. 98, 2008-2021.

Lauwereyns, J., Sakagami, M., Tsutsui, K., Kobayashi, S., Koizumi, M., Hikosaka, O., 2001. Responses to task-irrelevant visual features by primate prefrontal neurons. J. Neurophysiol. 86, 2001-2010.

Leung, H.-C., Gore, J.C., Goldman-Rakic, P.S., 2002. Sustained mnemonic response in the human middle frontal gyrus during on-line storage of spatial memoranda. J. Cog. Neurosci. 14, 659-671.

Levy, R., Goldman-Rakic, P.S., 1999. Association of storage and processing functions in the dorsolateral prefrontal cortex of the nonhuman primate. J. Neurosci. 19, $5149-5158$.

Levy, R., Goldman-Rakic, P.S., 2000. Segregation of working memory functions within the dorsolateral prefrontal cortex. Exp. Brain Res. 133, 23-32.

Ma, Y.-Y., Hu, X.-T., Wilson, F.A., 2012. The egocentric spatial reference frame used 
in dorsal-lateral prefrontal working memory in primates. Neurosci. Biobehav. Rev. $36,26-33$.

Mazzoni, P., Bracewell, R.M., Barash, S., Andersen, R.A., 1996. Spatially tuned auditory responses in area LIP of macaques performing delayed memory saccades to acoustic targets. J. Neurophysiol. 75, 1233-1241.

McCarthy, G., Blamire, A.M., Puce, A., Nobre, A.C., Bloch, G., Hyder, F., Goldman-Rakic, P.S., Shulman, R.G., 1994. Functional magnetic resonance imaging of human prefrontal cortex activation during a spatial working memory task. Proc. Natl. Acad. Sci. USA 91, 8690-8697.

McIntyre, J., Stratta, F., Lacquaniti, F., 1997. Viewer-centered frame of reference for pointing to memorized targets in three-dimensional space. J. Neurophysiol. 78, 1601-1618.

McIntyre, J., Stratta, F., Lacquaniti, F., 1998. Short-term memory for reaching to visual targets: psychophysical evidence for body-centered reference frames. J. Neurosci. 18, 8423-8435.

Messinger, A., Lebedev, M.A., Kralik, J.D., Wise, S.P., 2009. Multitasking of attention and memory functions in the primate prefrontal cortex. J. Neurosci. 29, 5640-5653.

Meyer, T., Qi, X.-L., Stanford, T.R., Constantinidis, C., 2011. Stimulus selectivity in dorsal and ventral prefrontal cortex after training in working memory tasks. J. Neurosci. 31, 6266-6276.

Mikami, A., Ito, S., Kubota, K., 1982. Visual response properties of dorsolateral prefrontal neurons during visual fixation task. J. Neurophysiol. 47, 593-605.

Miller, E.K., 2000. The prefrontal cortex and cognitive control. Nature Rev. Neurosci. 1, $59-65$.

Miller, E.K., Cohen, J.D., 2001. An integrative theory of prefrontal cortex function. Annu. Rev. Neurosci. 24, 167-202.

Mottaghy, F.M., Gangitano, M., Sparing, R., Krause, B.J., Pascual-Leone, A., 2002. 
Segregation of areas related to visual working memory in the prefrontal cortex revealed by rTMS. Cereb. Cortex 12, 369-375.

Mountcastle, V.B., Lynch, J.C., Georgopoulos, A., Sakata, H., Acuna, C., 1975. Posterior parietal association cortex of the monkey: command functions for operations within extrapersonal space. J. Neurophysiol. 38, 871-908.

Mushiake, H., Saito, N., Furusawa, Y., Izumiyama, M., Sakamoto, K., Shamoto, H., Shimizu, H., Yoshimoto, T., 2002. Orderly activations of human cortical areas during path-planning task. Neuroreport 13, 423-426.

Mushiake, H., Saito, N., Sakamoto, K., Itoyama, Y., and Tanji, J., 2006. Activity in the lateral prefrontal cortex reflects multiple steps of future events in action plans. Neuron 50, 631-641.

Mushiake, H., Saito, N., Sakamoto, K., Sato, Y., Tanji, J., 2001. Visually based path-planning by Japanese monkeys. Cognit. Brain Res. 11, 165-169.

Niki, H., 1974a. Differential activity of prefrontal units during right and left delayed response trials. Brain Res. 70, 346-349.

Niki, H., 1974b. Prefrontal unit activity during delayed alternation in the monkey. I. Relation to direction of response. Brain Res. 68, 185-196.

Niki, H., 1974c. Prefrontal unit activity during delayed alternation in the monkey. II. Relation to absolute versus relative direction of response. Brain Res. 68: 197-204.

Niki, H., Watanabe, M., 1976. Prefrontal unit activity and delayed response: relation to cue location versus direction of response. Brain Res. 105, 79-88.

Ninokura, Y., Mushiake, H., Tanji, J., 2003. Representation of the temporal order of visual objects in the primate lateral prefrontal cortex. J. Neurophysiol. 89, $2868-2873$

Ninokura, Y., Mushiake, H., Tanji, J., 2004. Integration of temporal order and object information in the monkey lateral prefrontal cortex. J. Neurophysiol. 91, 555-560.

Nystron, L.E., Braver, T.S., Sabb, F.W., Delgado, M.R., Noll, D.C., Cohen, J.D., 2000. 
Working memory for letters, shapes, and locations: fMRI evidence against stimulus-based regional organization in human prefrontal cortex. Neuroimage 11, 424-446.

O’Dhaniel, A.M.-G., Cohen, Y.E., Groh, J.M., 2005. Eye-centered, head-centered, and complex coding of visual and auditory targets in the intraparietal sulcus. J. Neurophysiol. 94, 2331-2352.

Olson, C.R., Gettner, S.N., 1995. Object-centered direction selectivity in the macaque supplementary eye field. Science 269, 985-988.

Olson, C.R., Gettner, S.N., 1999. Macaque SEF neurons encode object-centered directions of eye movements regardless of the visual attributes of instructional cues. J. Neurophysiol. 81, 2340-2346.

Olson, C.R., Tremblay, L. 2000. Macaque supplementary eye field neurons encode object-centered locations relative to both continuous and discontinuous objects. J. Neurophysiol. 83, 2392-2411.

O Scalaidhe, S.P., Wilson, F.A.W., Goldman.-Rakic, P.S., 1997. Areal segregation of face-processing neurons in prefrontal cortex. Science 278, 1135-1138.

O Scalaidhe, S.P., Wilson, F.A.W., Goldman-Rakic, P.S., 1999. Face-selective neurons during passive viewing and working memory performance of rhesus monkeys: evidence for intrinsic specialization of neuronal coding. Cereb. Cortex 9, 459-475.

Owen, A.M., Stern, C.E., Look, R.B., Tracey, I., Rosen, B.R., Petrides, M., 1998. Functional organization of spatial and nonspatial working memory processing within the human lateral frontal cortex. Proc. Natl. Acad. Sci. USA 95, 7721-7726.

Owen, A.M., Herrod, N.J., Menon, D.K., Clark, J.C., Downey, S.P.M.J., Carpenter, T.A., Minhas, P.S., Turkheimer, F.E., Williams, E.J., Robbins, T.W., Sahakian, B.J., Petrides, M., Pickard, J.D., 1999. Redefining the functional organization of working memory processes within human lateral prefrontal cortex. Eur. J. Neurosci. 11, $567-574$. 
Passingham, D., Sakai, K., 2004. The prefrontal cortex and working memory: physiology and brain imaging. Curr. Opin. Neurobiol. 14, 163-168.

Pertzov, Y., Avidan, G., Zohary, E., 2011. Multiple reference frames for saccadic planning in the human parietal cortex. J. Neurosci. 31, 1059-1068.

Petrides, M., 1994. Frontal lobes and working memory: evidence from investigations of the effects of cortical excisions in non-human primates. In: Boller, F., Spinnler, H., Hendler, J.A. (Eds.), Handbook of Neuropsychology, Vol. 9. Elsevier, Amsterdam, pp.59-82.

Petrides, M., Pandya, D.N., 1984. Projections to the frontal cortex from the posterior parietal region in the rhesus monkey. J. Comp. Neurol. 228, 105-116.

Petrides, M., Pandya, D.N., 1994. Comparative architectonic analysis of the human and the macaque frontal cortex. In: Boller, F., Spinnler, H., Hendler, J.A. (Eds.), Handbook of Neuropsychology, Vol. 9. Elsevier, Amsterdam, pp.17-58.

Pigarev, I.N., Rizzolatti, G., Scandolara, C., 1979. Neurons responding to visual stimuli in the frontal lobe of macaque monkeys. Neurosci. Lett. 12, 207-212.

Postle, B.R., Berger, J.S., Taich, A.M., D’Esposito, M., 2000a. Activity in human frontal cortex associated with spatial working memory and saccadic behavior. J. Cogn. Neurosci. 12 (Suppl. 2), 2-14.

Postle, B.R., Stern, C.E., Rosen, B.R., Corkin, S., 2000b. An fMRI investigation of cortical contributions to spatial and nonspatial visual working memory. Neuroimage $11,409-423$.

Procyk, E., Goldman-Rakic, P.S., 2006. Modulation of dorsolateral prefrontal delay activity during self-organized behavior. J. Neurosci. 26, 11313-11323.

Quintana, J., Fuster, J.M., 1999. From perception to action: temporal integrative functions of prefrontal and parietal neurons. Cereb. Cortex 9, 213-221.

Quintana, J., Yajeya, J., Fuster, J.M., 1988. Prefrontal representation of stimulus attributes during delay tasks. I. Unit activity in cross-temporal integration of sensory 
and sensory-motor information. Brain Res. 474, 211-221.

Rainer, G., Asaad, W.F., Miller, E.K., 1998a. Selective representation of relevant information by neurons in the primate prefrontal cortex. Nature 393, 577-579.

Rainer, G., Asaad, W.F., Miller, E.K., 1998b. Memory fields of neurons in the primate prefrontal cortex. Proc. Natl. Acad. Sci. USA 95, 15008-15013.

Rainer, G., Rao, S.C., Miller, E.K., 1999. Prospective coding for objects in primate prefrontal cortex. J. Neurosci. 19, 5493-5505.

Rao, S.C., Rainer, G., Miller, E.K., 1997. Integration of what and where in the primate prefrontal cortex. Science 276, 821-824.

Rao, S.G., Williams, G.V., Goldman-Rakic, P.S., 1999. Isodirectional tuning of adjacent interneurons and pyramidal cells during working memory: evidence for microcolumnar organization in PFC. J. Neurophysiol. 81, 1903-1916.

Rao, S.G., Williams, G.V., Goldman-Rakic, P.S., 2000. Destruction and creation of spatial tuning by disinhibition: $\mathrm{GABA}_{\mathrm{A}}$ blockade of prefrontal cortical neurons engaged by working memory. J. Neurosci. 20, 485-494.

Renart, A., Song, P., Wang, X.-J., 2003. Robust spatial working memory through homeostatic synaptic scaling in heterogeneous cortical networks. Neuron 38, 473-485.

Reppas, J.B., Niyogi, S., Dala, A.M., Sereno, M.I., Tootell, R.B.H., 1997. Representation of motion boundaries in retinotopic human visual cortical areas. Nature 388, 175-179.

Rizzolatti, G., Scandolara, C., Matelli, M., Gentilucci, M., 1981. Afferent properties of periarcuate neurons in macaque monkeys. II. Visual responses. Behav. Brain Res. 2, 147-163.

Rizzuto, D.S., Mamelak, A.N., Sutherling, W.W., Fineman, I., Andersen, R.A., 2005. Spatial selectivity in human ventrolateral prefrontal cortex. Nature Neurosci. 8, 415-417. 
Robertson, E.M., Tormos, J.M., Maeda, F., Pascual-Leone, A., 2001. The role of the dorsolateral prefrontal cortex during sequence learning is specific for spatial information. Cereb. Cortex 11, 628-635.

Romanski, L.M., Averbeck, B.B., 2009. The primate cortical auditory system and neural representation of conspecific vocalizations. Annu. Rev. Neurosci. 32, 315-346.

Romanski, L.M., Averbeck, B.B., Diltz, M., 2004. Neural representation of vocalizations in the primate ventrolateral prefrontal cortex. J. Neurophysiol. 93, 734-747.

Romanski, L.M., Goldman-Rakic, P.S., 2001. An auditory domain in primate prefrontal cortex. Nature Neurosci. 5, 15-16.

Romanski, L.M., Bates, J.F., Goldman-Rakic, P.S., 1999a. Auditory belt and parabelt projections to the prefrontal cortex in the rhesus monkey. J. Comp. Neurol. 403, 141-157.

Romanski, L.M., Tian, B., Fritz, J., Mishkin, M., Goldman-Rakic, P.S., Rauschecker, J.P., 1999b. Dual streams of auditory afferents target multiple domains in the primate prefrontal cortex. Nature Neurosci. 2, 1131-1136.

Rosenbluth, D., Allman, J.M., 2002. The effect of gaze angle and fixation distance on the responses of neurons in V1, V2, and V4. Neuron 33, 143-148.

Rosenkilde, C.E., 1979. Functional heterogeneity of the prefrontal cortex in the monkey: a review. Behav. Neural Biol. 25, 301-345.

Rosenkilde, C.E., Bauer, R.H., Fuster, J.M., 1981. Single cell activity in ventral prefrontal cortex of behaving monkeys. Brain Res. 209, 375-394.

Rowe, J.B., Sakai, K., Lund, T.E., Ramsoy, T., Christensen, M.S., Baare, W.F.C., Paulson, O.B., Passingham, R.E., 2007. Is the prefrontal cortex necessary for establishing cognitive sets? J. Neurosci. 27, 13303-13310.

Rowe, J.B., Toni, I., Josephs, O., Frackowiak, R.S.J., Passingham, R.E., 2000. The 
prefrontal cortex: response selection or maintenance within working memory? Science 288, 1656-1660.

Rushworth, M.F.S., Nixon, P.D., Eacott, M.J., Passingham, R.E., 1997. Ventral prefrontal cortex is not essential for working memory. J. Neurosci. 17, 4829-4838.

Saito, N., Mushiake, H., Sakamoto, K., Itoyama, Y., Tanji, J., 2005. Representation of immediate and final behavioral goals in the monkey prefrontal cortex during an instructed delay period. Cereb. Cortex 15, 1535-1546.

Sakagami, M., Niki, H., 1994. Spatial selectivity of go/no-go neurons in monkey prefrontal cortex. Exp. Brain Res. 100, 165-169.

Sakagami, M., Tsutsui, K., 1999. The hierarchical organization of decision making in the primate prefrontal cortex. Neurosci. Res. 34, 79-89.

Sakata, H., Taira, M., Kusunoki, M., Murata, A., Tanaka, Y., 1997. The parietal association cortex in depth perception and visual control of hand action. Trends. Neurosci. 20, 350-357.

Sala, J.B., Rama, P., Courtney, S.M., 2003. Functional topography of a distributed neural system for spatial and nonspatial information maintenance in working memory. Neuropsychologia 41, 341-356.

Sawaguchi, T., 1987. Properties of neuronal activity related to a visual reaction time task in the monkey prefrontal cortex. J. Neurophysiol. 58, 1080-1099.

Sawaguchi, T., 1998. Attenuation of delay-period activity of monkey prefrontal neurons by an $\alpha_{2}$-adrenergic antagonist during an oculomotor delayed-response task. J. Neurophysiol. 80, 2200-2205.

Sawaguchi, T., Iba, M., 2001. Prefrontal cortical representation of visuospatial working memory in monkeys examined by local inactivation with muscimol. J. Neurophysiol. $86,2041-2053$.

Sawaguchi, T., Matsumura, M., Kubota, K., 1989. Depth distribution of neuronal activity related to a visual reaction time task in the monkey prefrontal cortex. J. 
Neurophysiol. 61, 435-446.

Sawaguchi, T., Yamane, I. 1999. Properties of delay-period neuronal activity in the monkey dorsolateral prefrontal cortex during a spatial delayed matching-to-sample task. J. Neurophysiol. 82, 2070-2080.

Schlicht, E.J., Schrater, P.R., 2007. Impact of coordinate transformation uncertainty on human sensorimotor control. J. Neurophysiol. 97, 4203-4214.

Schwartz, M.L., Goldman-Rakic, P.S., 1984. Callosal and intrahemispheric connectivity of the prefrontal association cortex in rhesus monkey: relation between intraparietal and principal sulcal cortex. J. Comp. Neurol. 226, 403-420.

Seltzer, B., Pandya, D.N., 1989. Frontal lobe connections of the superior temporal sulcus in the rhesus monkey. J. Comp. Neurol. 281, 97-113.

Sereno, M.I., Pitzalis, S., Martinez, A., 2001. Mapping of contralateral space in retinotopic coordinates by a parietal cortical area in humans. Science 294, $1350-1354$.

Silver, M.A., Ress, D., Heeger, D.J., 2005. Topographic maps of visual spatial attention in human parietal cortex. J. Neurophysiol. 94, 1358-1371.

Spiers, H.J., 2008. Keeping the goal in mind: prefrontal contributions to spatial navigation. Neuropsychologia 46, 2106-2108.

Suzuki, H., 1985. Distribution and organization of visual and auditory neurons in the monkey prefrontal cortex. Vision Res. 25, 465-469.

Suzuki, H., Azuma, M., 1977. Prefrontal neuronal activity during gazing at a light spot in the monkey. Brain Res. 126, 497-508.

Suzuki, H., Azuma, M., 1983. Topographic studies on visual neurons in the dorsolateral prefrontal cortex of the monkey. Exp. Brain Res. 53, 47-58.

Suzuki, H., Azuma, M., Yumiya, H., 1979. Stimulus and behavioral factors contributing to the activation of monkey prefrontal neurons during gazing. Jap. J. Physiol. 29, 471-489. 
Takeda, K., Funahashi, S., 2002. Prefrontal task-related activity representing visual cue location or saccade direction in spatial working memory tasks. J. Neurophysiol. 87, $567-588$.

Takeda, K., Funahashi, S., 2004. Population vector analysis of primate prefrontal activity during spatial working memory. Cereb. Cortex 14, 1328-1339.

Takeda, K., Funahashi, S., 2007. Relationship between prefrontal task-related activity and information flow during spatial working memory performance. Cortex 43, $38-52$.

Tanila, H., Carlson, S., Linnankoski, I., Kahila, H., 1993. Regional distribution of functions in dorsolateral prefrontal cortex of the monkey. Behav. Brain Res. 53, $63-71$.

Tanila, H., Carlson, S., Linnankoski, I., Lindroos, F., Kahila, H., 1992. Functional properties of dorsolateral prefrontal cortical neurons in awake monkey. Behav. Brain Res. 47, 169-180.

Ungerleider, L.G., Gaffan, D., Pelak, V.S., 1989. Projections from inferior temporal cortex to prefrontal cortex via the uncinate fascicle in rhesus monkeys. Exp. Brain Res. 76, 473-484.

Ungerleider, L.G., Mishkin, M., 1982. Two cortical visual systems. In: Engle, D.J., Goodale, M.A., Mansfield, R.J. (Eds.), Analysis of Visual Behavior. MIT Press, Cambridge, MA, pp.549-586.

Vaadia, E., Benson, D.A., Hienz, R.D., Goldstein, Jr., M.H., 1986. Unit study of monkey frontal cortex: active localization of auditory and of visual stimuli. J. Neurophysiol. 56, 934-952.

Vallar, G., Lobel, E., Galati, G., Berthoz, A., Pizzamiglio, L., Le Bihan, D., 1999. A fronto-parietal system for computing the egocentric spatial frame of reference in humans. Exp. Brain Res. 124, 281-286.

Vijayraghavan, S., Wang, M., Birnbaum, S., Williams, G.V., Arnsten, A.F.T., 2007. 
Inverted-U dopamine D1 receptor actions on prefrontal neurons engaged in working memory. Nature Neurosci. 10, 376-384.

Wallis, J.D., Anderson, K.C., Miller, E.K., 2001. Single neurons in prefrontal cortex encode abstract rules. Nature 411, 953-956.

Wang, M., Vijayraghavan, S., Goldman-Rakic, P.S., 2004. Selective D2 receptor actions on the functional circuitry of working memory. Science 303, 853-856.

Watanabe, K., Funahashi, S., 2007. Prefrontal delay-period activity reflects the decision process of a saccade direction during a free-choice ODR task. Cereb. Cortex 17, i88-i100.

Watanabe, K., Igaki, S., Funahashi, S., 2006. Contributions of prefrontal cue-, delay-, and response-period activity to the decision process of saccade direction in a free-choice ODR task. Neural Networks 19 1203-1222.

Watanabe, M., 1986a. Prefrontal unit activity during delayed conditional go/no-go discrimination in the monkey. I. relation to the stimulus. Brain Res. 382, 1-14.

Watanabe, M., 1986b. Prefrontal unit activity during delayed conditional go/no-go discrimination in the monkey. II. Relation to go and no-go responses. Brain Res. $382,15-27$.

Watanabe, M., 1990. Prefrontal unit activity during associative learning in the monkey. Exp. Brain Res. 80, 296-309.

Watanabe, Y., Funahashi, S., 2004a. Neuronal activity throughout the primate mediodorsal nucleus of the thalamus during oculomotor delayed-responses. I. Cue-, delay-, and response-period activity. J. Neurophysiol. 92, 1738-1755.

Watanabe, Y., Funahashi, S., 2004b. Neuronal activity throughout the primate mediodorsal nucleus of the thalamus during oculomotor delayed-responses. II. Activity encoding visual versus motor signal. J. Neurophysiol. 92, 1756-1769.

Watanabe, Y., Funahashi, S., 2012. Thalamic mediodorsal nucleus and working memory. Neurosci. Biobehav. Rev. 36, 134-142. 
White, I.M., Wise, S.P., 1999. Rule-dependent neuronal activity in the prefrontal cortex. Exp. Brain Res. 126, 315-335.

Williams, G.V., Goldman-Rakic, P.S., 1995. Modulation of memory fields by dopamine D1 receptors in prefrontal cortex. Nature 376, 572-575.

Wilson, F.A.W., O Scalaidhe, S.P., Goldman-Rakic, P.S., 1993. Dissociation of object and spatial processing domains in primate prefrontal cortex. Science 260, 1955-1958.

Wilson, F.A.W., O Scalaidhe, S.P., Goldman-Rakic, P.S., 1994. Functional synergism between putative $\gamma$-aminobutyrate-containing neurons and pyramidal neurons in prefrontal cortex. Proc. Natl. Acad. Sci. USA 91, 4009-4013.

Yajeya, J., Quintana, J., Fuster, J.M., 1988. Prefrontal representation of stimulus attributes during delay tasks. II. The role of behavioral significance. Brain Res. 474, 222-230.

Yoshida, W., Ishii, S., 2006. Resolution of uncertainty in prefrontal cortex. Neuron 50, 781-789.

Zhang, T., Heuer, H.W., Britten, K.H., 2004. Parietal area VIP neuronal responses to heading stimuli are encoded in head-centered coordinates. Neuron 42, 993-1001. 


\section{Figure legends}

Figure 1: A. A schematic drawing of the experimental condition using the Wisconsin general test apparatus (WGTA). B. Delayed-response performance in the WGTA. After Goldman-Rakic (1987).

Figure 2: A. Schematic drawings of the temporal sequence of the oculomotor delayed-response (ODR) task. The monkey faced a computer monitor on which a fixation target (a small white spot) and a visual cue (a white square) were presented. B. Three typical examples of task-related activities observed in the ODR task: cue-period activity (upper figure), delay-period activity (middle figure), and response-period activity (lower figure). C, D, and R indicate the cue period ( $0.5 \mathrm{~s})$, delay period (3.0 s), and response period ( $\max 0.5 \mathrm{~s})$, respectively. After Goldman-Rakic et al. (1990).

Figure 3: A. Schematic drawing of the path-finding task performed by monkeys. B. Three combinations of the directions of wrist movements and the directions of cursor movement on the monitor. The direction of each arrow indicates the direction of cursor movement on the monitor. After Mushiake et al. (2006).

Figure 4: Topographic representations of the size of the visual receptive field (left figures) and the eccentricity of the center of the visual receptive field (right figures) in three monkeys (A, B, and C). Neurons located in the lateral prefrontal cortex and close to the inferior arcuate sulcus had smaller receptive fields and represented the foveal and parafoveal regions, while those in the medial part of the prefrontal cortex had larger receptive fields and represented a more eccentric position from the fovea. The size of the receptive field gradually increased for neurons located anteriorly and this tendency was observed without a change in the eccentricity of the receptive field center. After 
Suzuki and Azuma (1983).

Figure 5: A. Schematic drawings of the temporal sequences of the oculomotor delayed-response (ODR) task and the rotatory ODR (R-ODR) task. In the ODR task, the monkey was required to make a saccade toward the position where the visual cue was presented, while in the R-ODR task, the monkey was required to make a saccade toward the direction $90^{\circ}$ clockwise from the direction of the visual cue. B. Arrangements of the visual cue locations in the ODR task and the R-ODR task.

Figure 6: An example of delay-period activity representing the direction of the visual cue. A. Delay-period activity observed in the ODR task. Significant delay-period activity was observed when the visual cue was presented at the $270^{\circ}$ and $315^{\circ}$ directions. B. The same neuron's delay-period activity in the R-ODR task. Significant delay-period activity was observed when the visual cue was presented at the $270^{\circ}$ direction. In this trial, the monkey was required to make a saccade toward the $180^{\circ}$ direction. Therefore, this delay-period activity is believed to represent the direction of the visual cue, rather than the direction of the saccade. C. Two other examples of delay-period activity representing the direction of the visual cue. The difference between the peaks of the tuning curves calculated from the mean discharge rates of delay-period activity between the two tasks was less than $45^{\circ} . \mathrm{C}, \mathrm{D}$, and $\mathrm{R}$ indicate the cue period, delay period, and response period, respectively. The cue period and delay period were $0.5 \mathrm{~s}$ and $3.0 \mathrm{~s}$, respectively. Rasters and histograms were arranged based on the cue directions. After Takeda and Funahashi (2002).

Figure 7: An example of delay-period activity representing the direction of the saccade. A. Delay-period activity observed in the ODR task. Significant delay-period activity was observed when the visual cue was presented at the $0^{\circ}$ direction. B. The same 
neuron's delay-period activity in the R-ODR task. Significant delay-period activity was observed when the visual cue was presented at the $90^{\circ}$ direction. In this trial, the monkey was required to make a saccade toward the $0^{\circ}$ direction. Therefore, this delay-period activity is believed to represent the direction of the saccade, rather than the direction of the visual cue. C. The distribution of the difference in the best directions of delay-period activity between the ODR and R-ODR tasks. Most of the neurons were distributed within $45^{\circ}$, indicating that most delay-period activity represented the direction of the visual cue. After Takeda and Funahashi (2002).

Figure 8: An example of delay-period activity that represents the relative spatial position of the visual cue. A. The experimental arrangement of the fixation target and visual cues to examine whether delay-period activity represented relative or absolute spatial positions on the monitor. B. An example of delay-period activity observed in the prefrontal cortex. This neuron exhibited significant delay-period activity when the visual cue was presented on the left side of the fixation target. Significant delay-period activity was observed when the visual cue was presented on the left side in the right fixation condition, but no delay-period activity was observed when the visual cue was presented on the right side in the central fixation condition (red squares). Similarly, significant delay-period activity was observed when the visual cue was presented on the left side in the central fixation condition, but no delay-period activity was observed when the visual cue was presented on the right side in the left fixation condition (green squares). Thus, this result indicates that this directional delay-period activity does not encode the absolute spatial position of the visual cue in the visual field, but rather encodes the spatial position of the visual cue relative to the fixation target. After Funahashi and Takeda (2002).

Figure 9: Population vector analysis using directional task-related activities observed in 
the ODR and R-ODR tasks. A. Temporal change in population vectors during the $180^{\circ}$ trial in the ODR task. Population vectors were calculated using the activities of 121 neurons during a 250-ms time-window that was moved in $50 \mathrm{~ms}$ time-steps from the onset of the visual cue until the end of the response period. All population vectors were in the same direction as the visual cue. B. Temporal changes in the difference between the directions of population vectors and the cue directions in the ODR task. Each data point represents the mean difference and its standard error between the direction of the population vector and the cue direction. The mean differences were close to $0^{\circ}$ throughout the delay period. C. Temporal change in population vectors along the $180^{\circ}$ trial of the R-ODR task. The population vectors were calculated using the same method as was used for the ODR task. (B) Temporal changes in the difference between the directions of population vectors and the cue directions in the R-ODR task. The mean differences changed gradually from close to $0^{\circ}$ to almost $90^{\circ}$ during the delay period. After Takeda and Funahashi (2004).

Figure 10: Schematic drawings of temporal profiles of task-related activity for six groups of prefrontal neurons. C, D, and R indicate cue-period activity, delay-period activity, and response-period activity, respectively. cue and sac indicate activity that represents the cue direction and the saccade direction, respectively. After Takeda and Funahashi (2007).

Figure 11: A schematic diagram of information flow during delayed-response performance based on what kind of task-related activity prefrontal neurons exhibited and what information this activity represented. C, D, and R indicate cue-period activity, delay-period activity, and response-period activity, respectively. cue and sac indicate activity that represents the cue direction and the saccade direction, respectively. MD indicates the mediodorsal nucleus of the thalamus. The MD might provide saccade 
information to the prefrontal cortex, and this might help to generate delay-period activity that represents prospective saccade information. After Watanabe and Funahashi (2012). 


\section{A. Wisconsin general test apparatus (WGTA) \\ B. Delayed-response task}
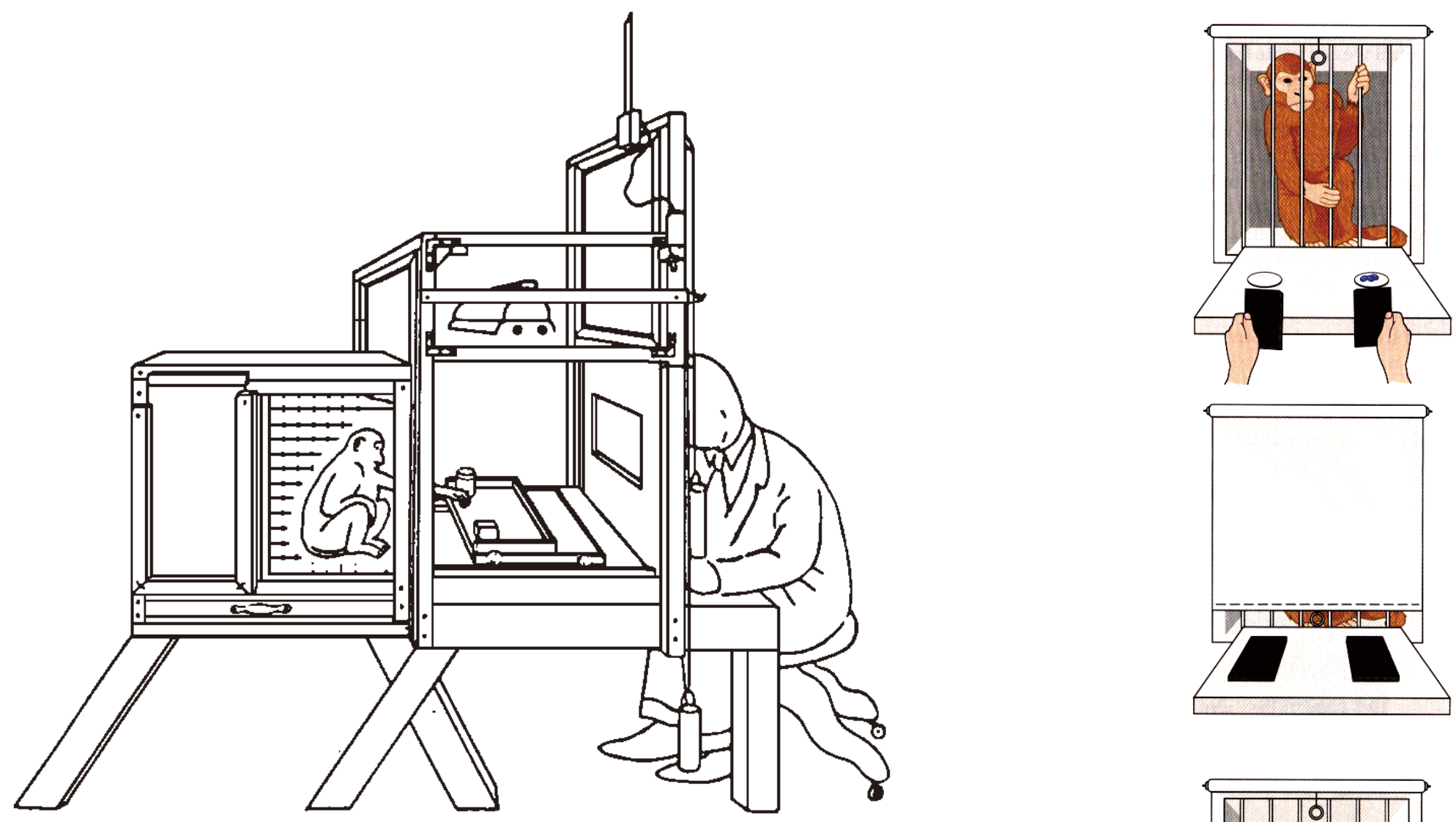

Cue period

Delay period

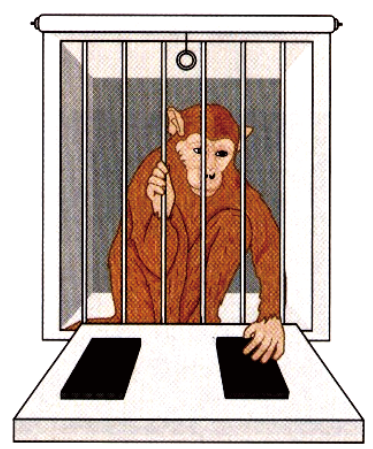

Response period 


\section{A. Oculomotor delayed-response (ODR) task B. Activity observed in ODR task}
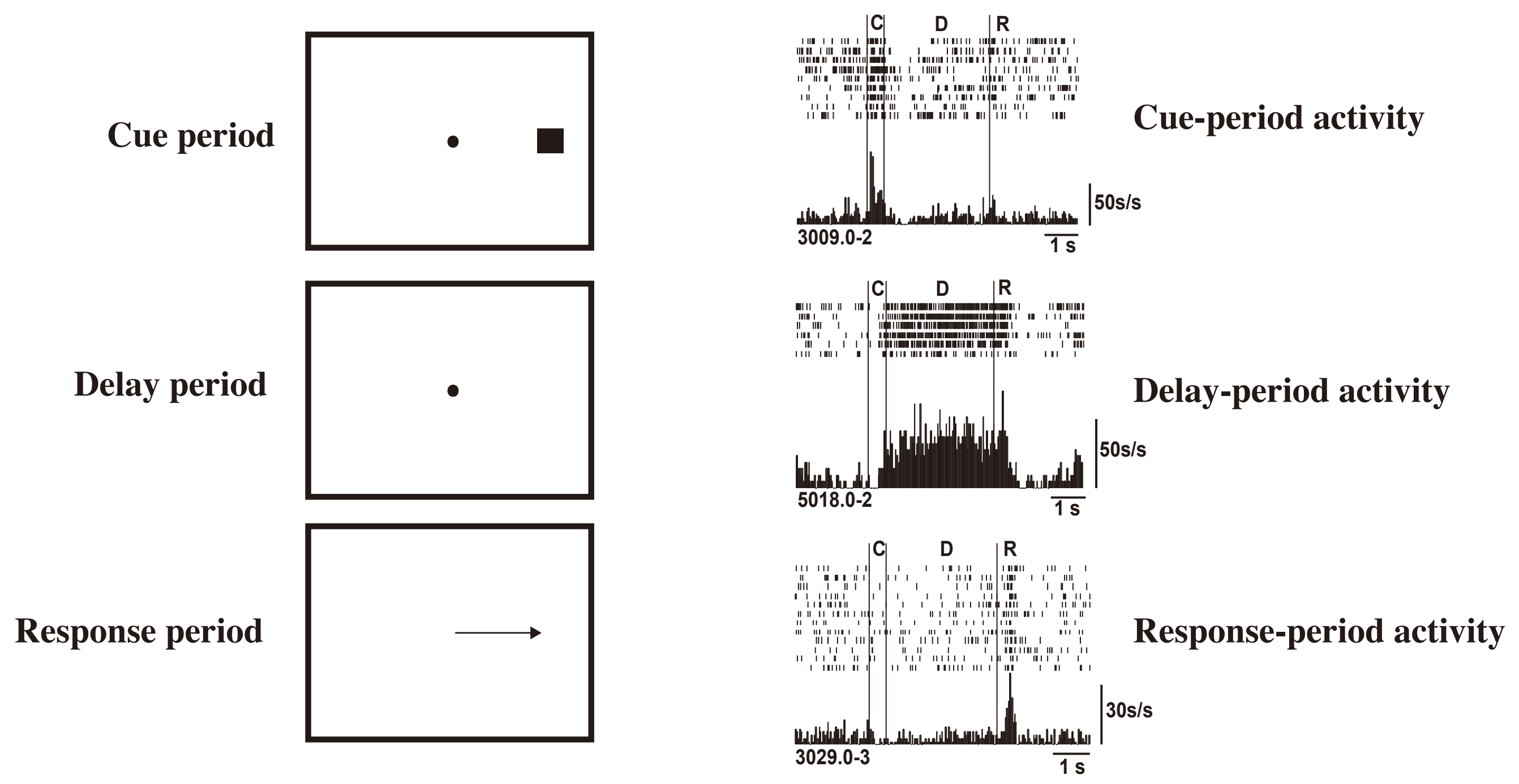
A

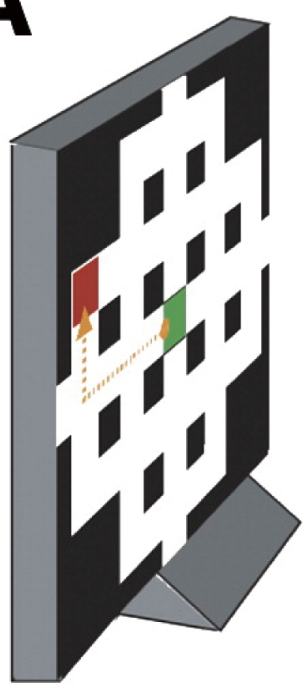

B

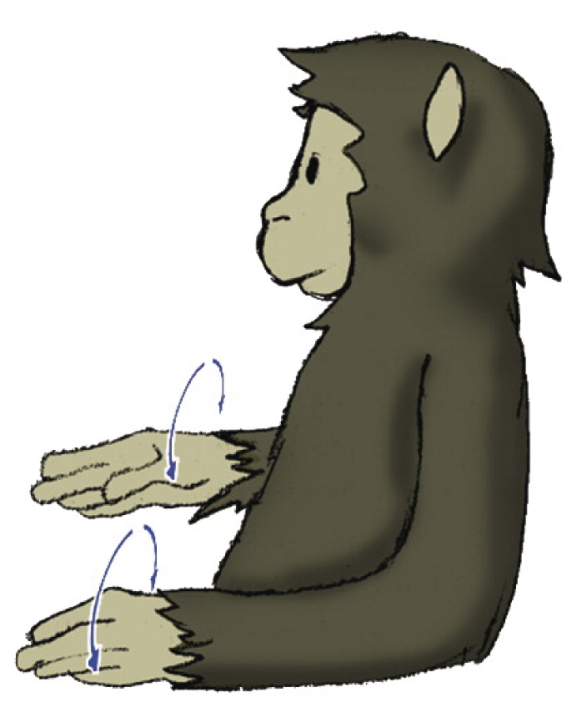

Right Hand Supination Pronation Pronation Supination

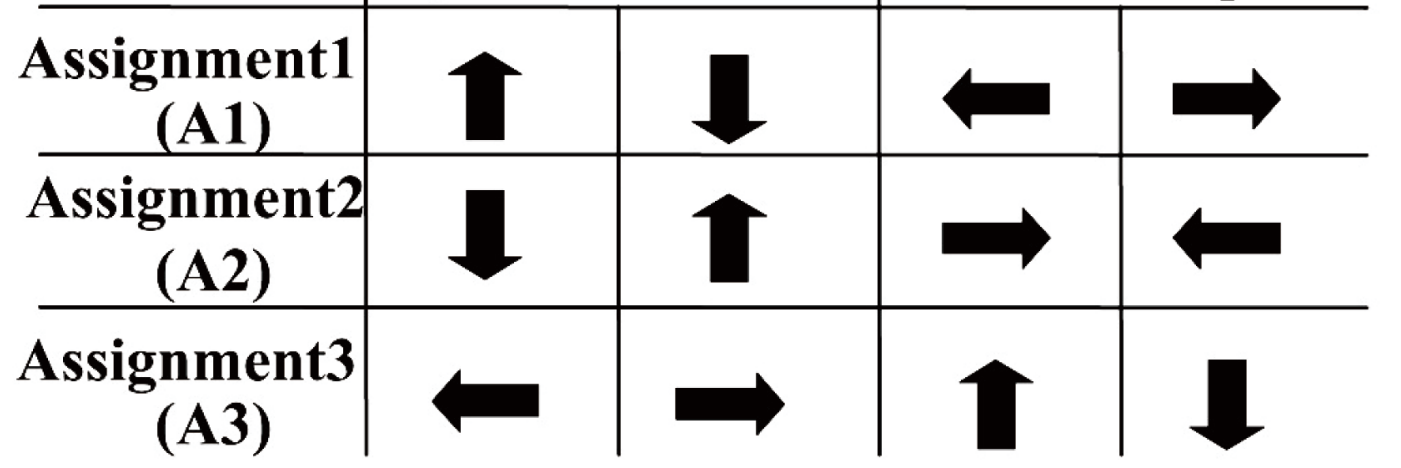




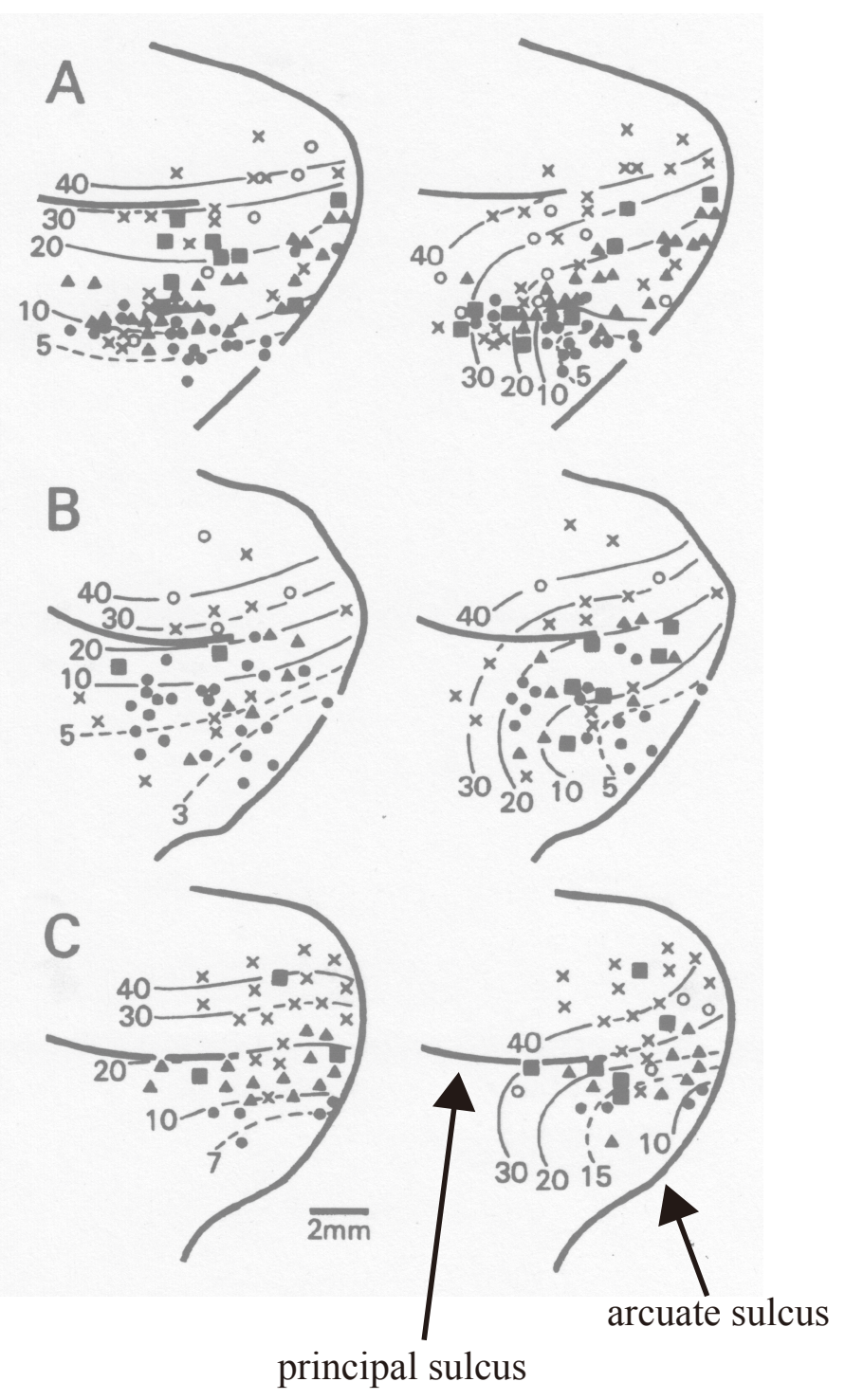


A. Temporal sequence of ODR and R-ODR tasks

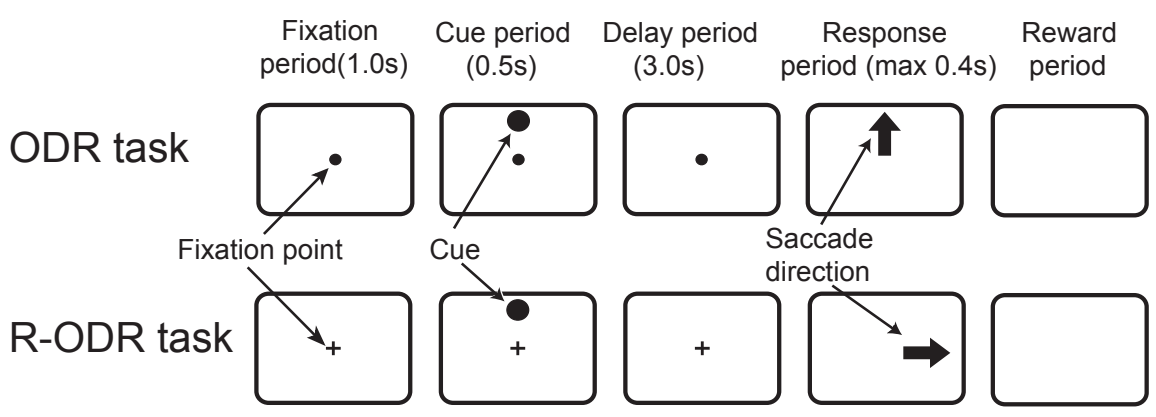

B. Arrangement of the visual cue in two tasks

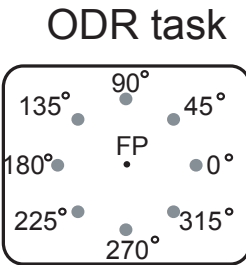

R-ODR task

\begin{tabular}{|ccc|}
\hline & $90^{\circ}$ & \\
$180^{\circ} \cdot$ & $\stackrel{F P}{+}$ & \\
& & \\
& & \\
& $270^{\circ}$ & \\
\hline
\end{tabular}




\section{A. Activity of neuron $\mathrm{m070}$ in ODR task}

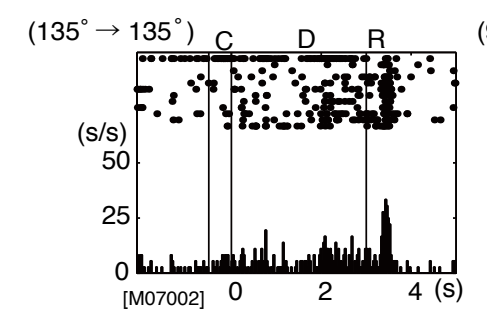

$\left(180^{\circ} \rightarrow 180^{\circ}\right)$ C $\quad$ D $\quad R$
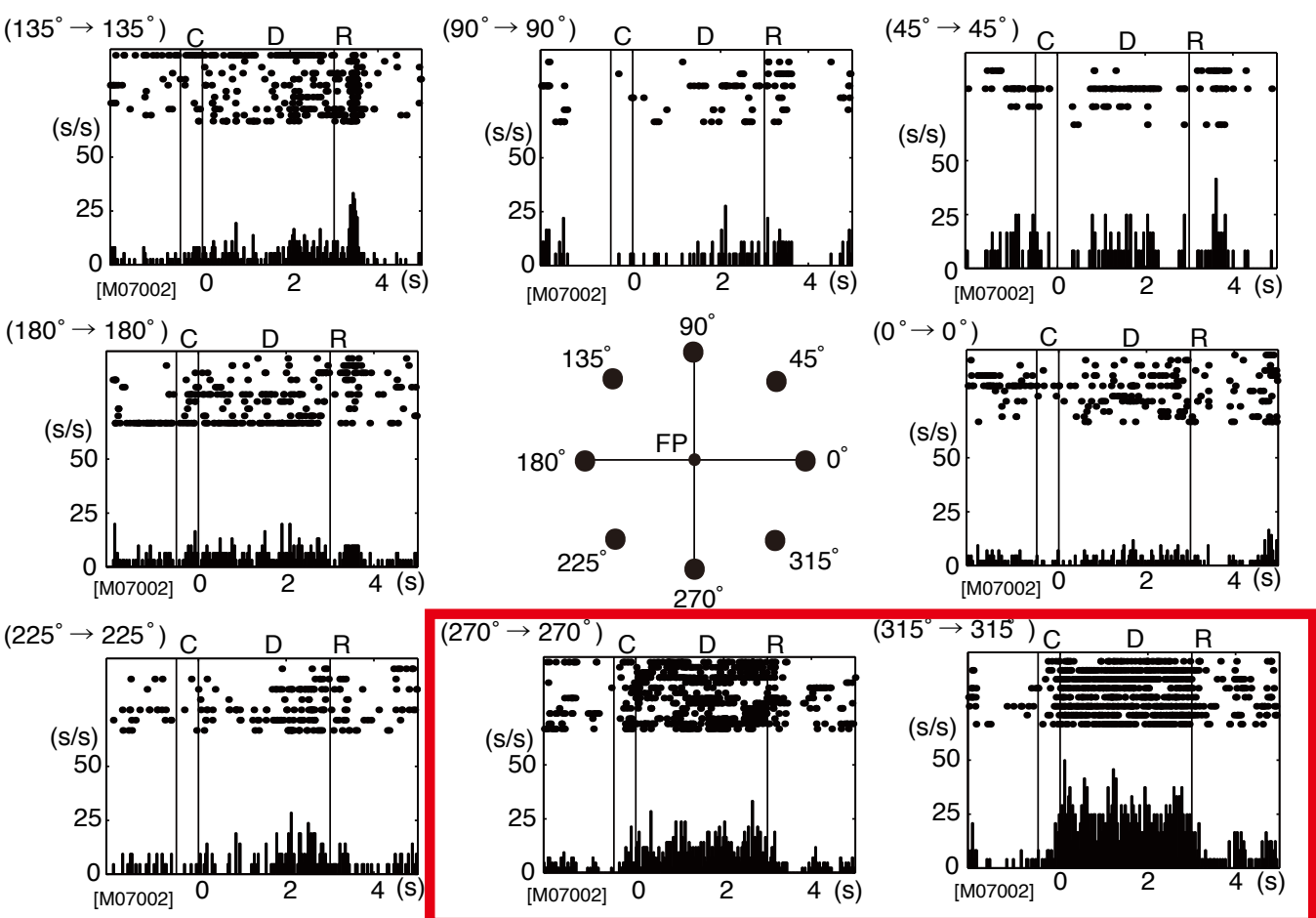

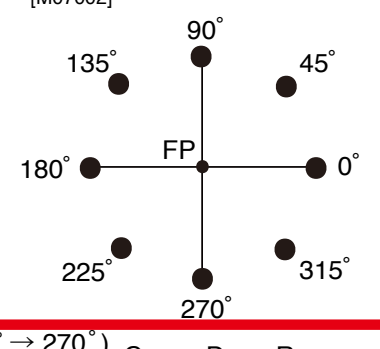

B. Activity of neuron m070 in R-ODR task
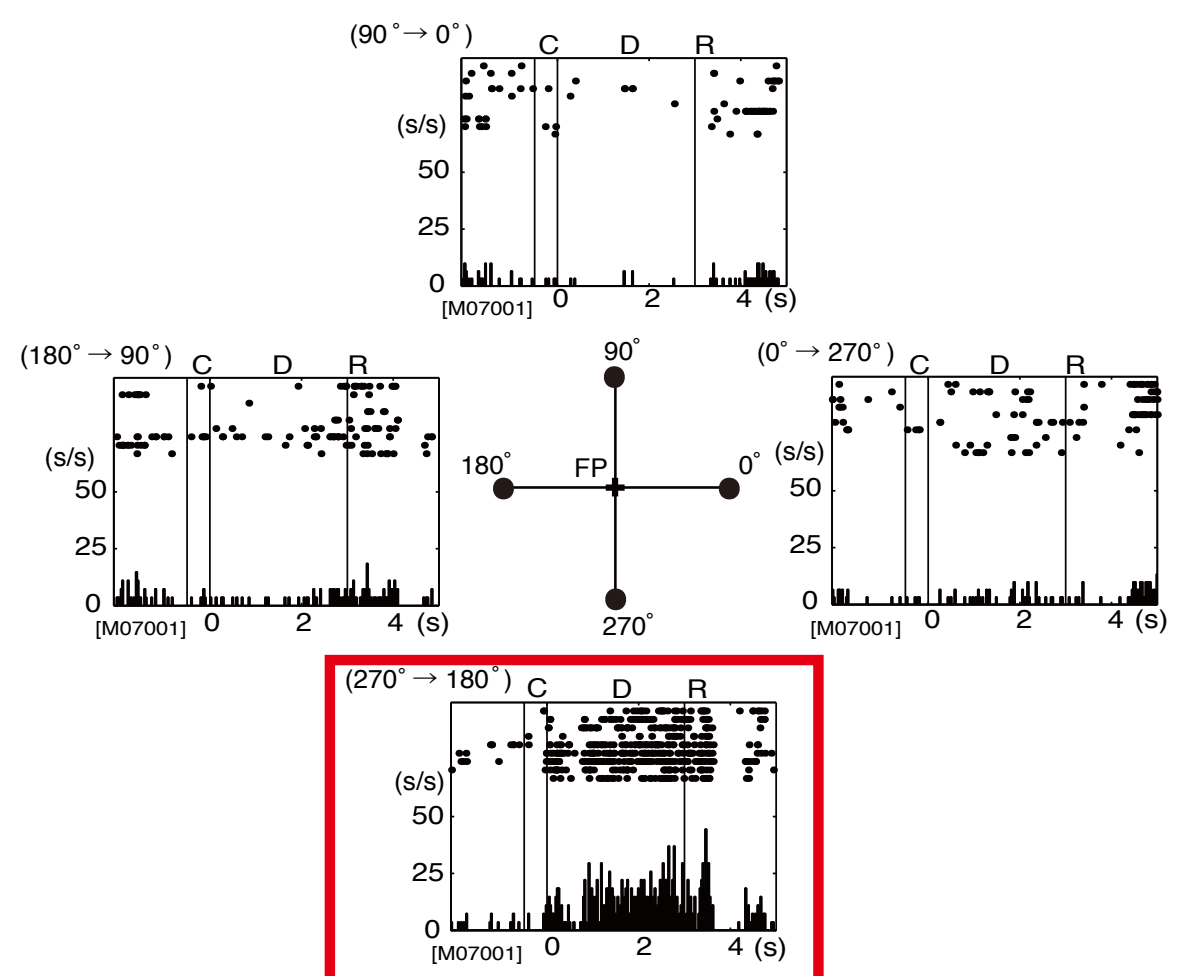

C. Two examples of tuning curves of delay-period activity
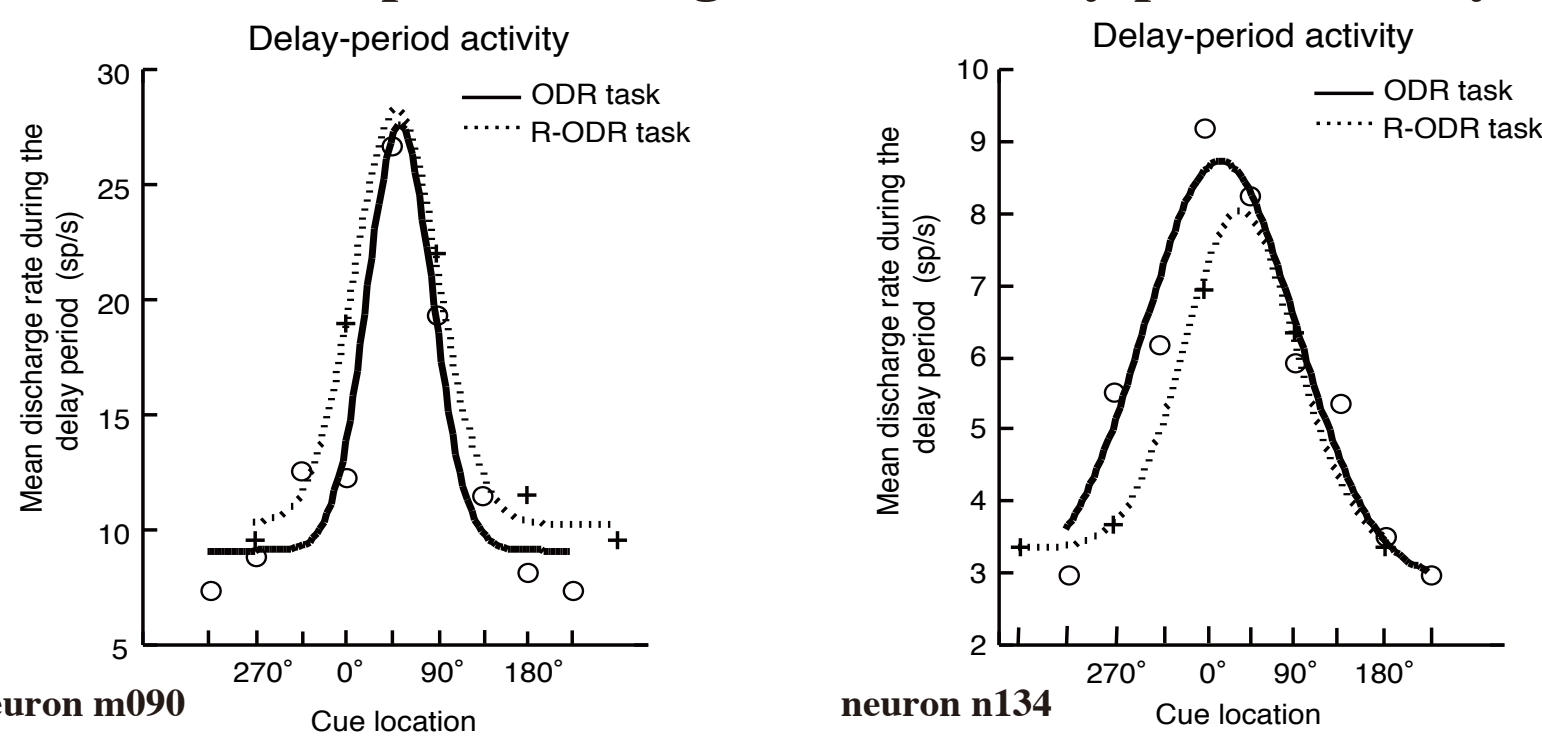
A. Activity of neuron m072 in ODR task
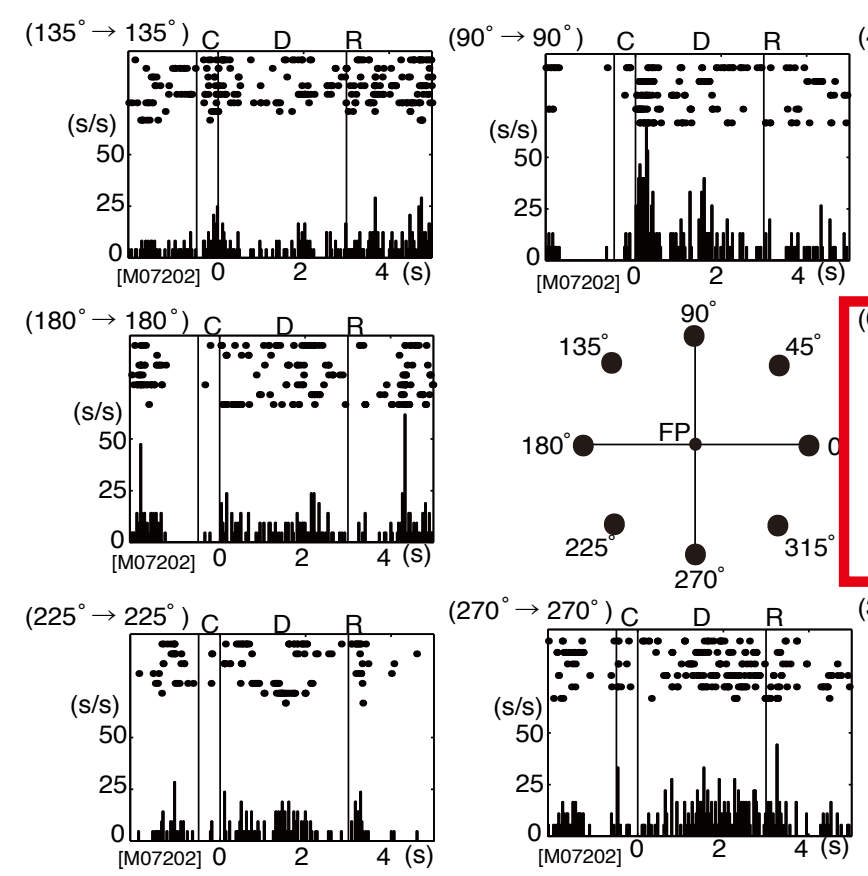

B. Activity of neuron m072 in R-ODR task

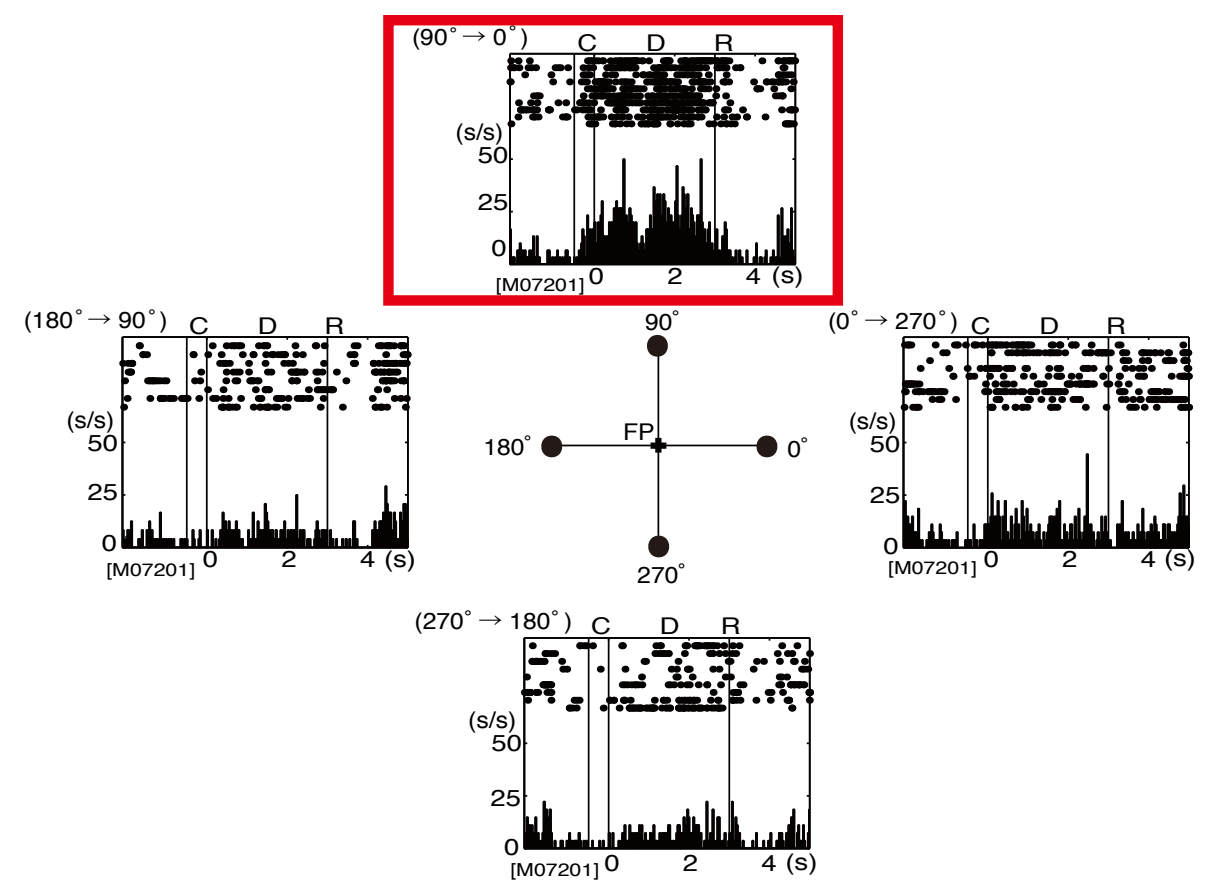

C. Difference of the best directions of delay-period activity between ODR and R-ODR tasks

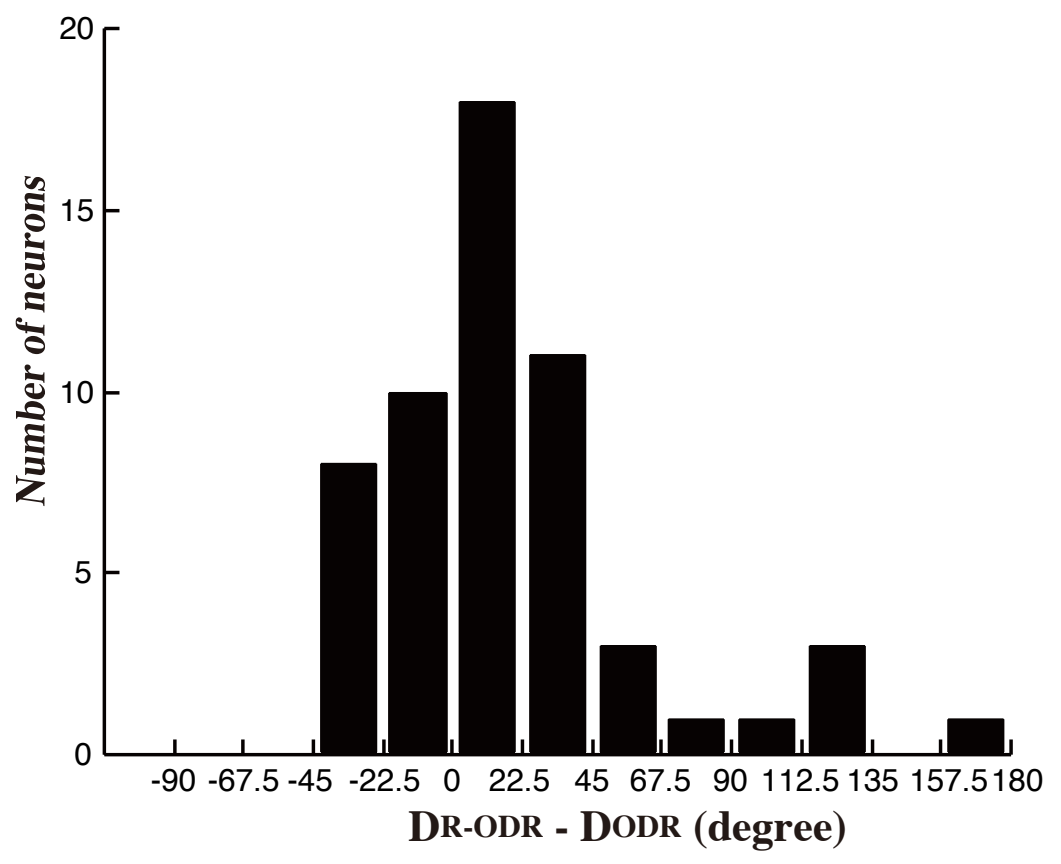



A. ODR task $\left(180^{\circ}\right.$ trial $)$
B. ODR task
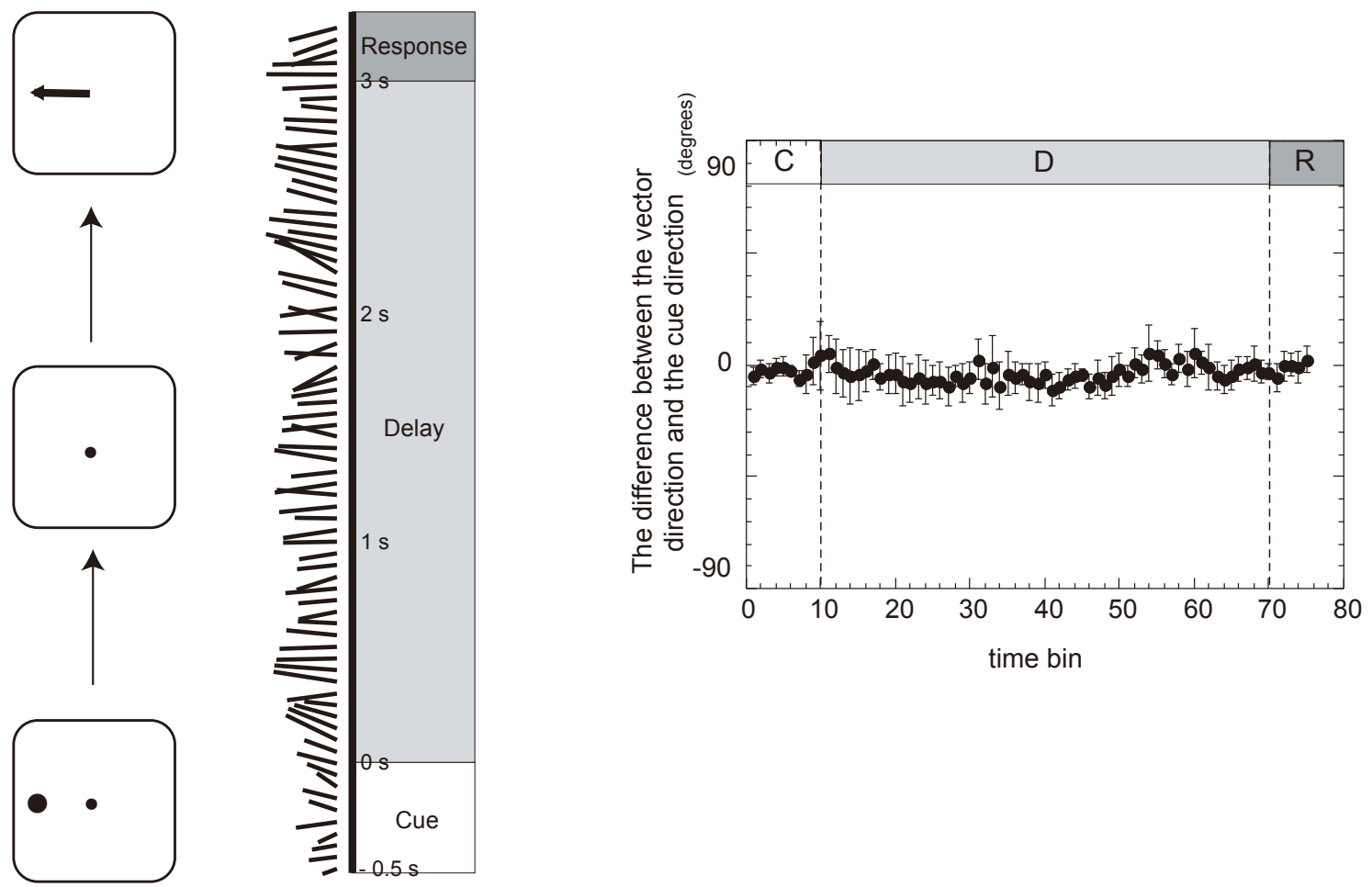

C. R-ODR task $\left(180^{\circ}\right.$ trial)

D. R-ODR task
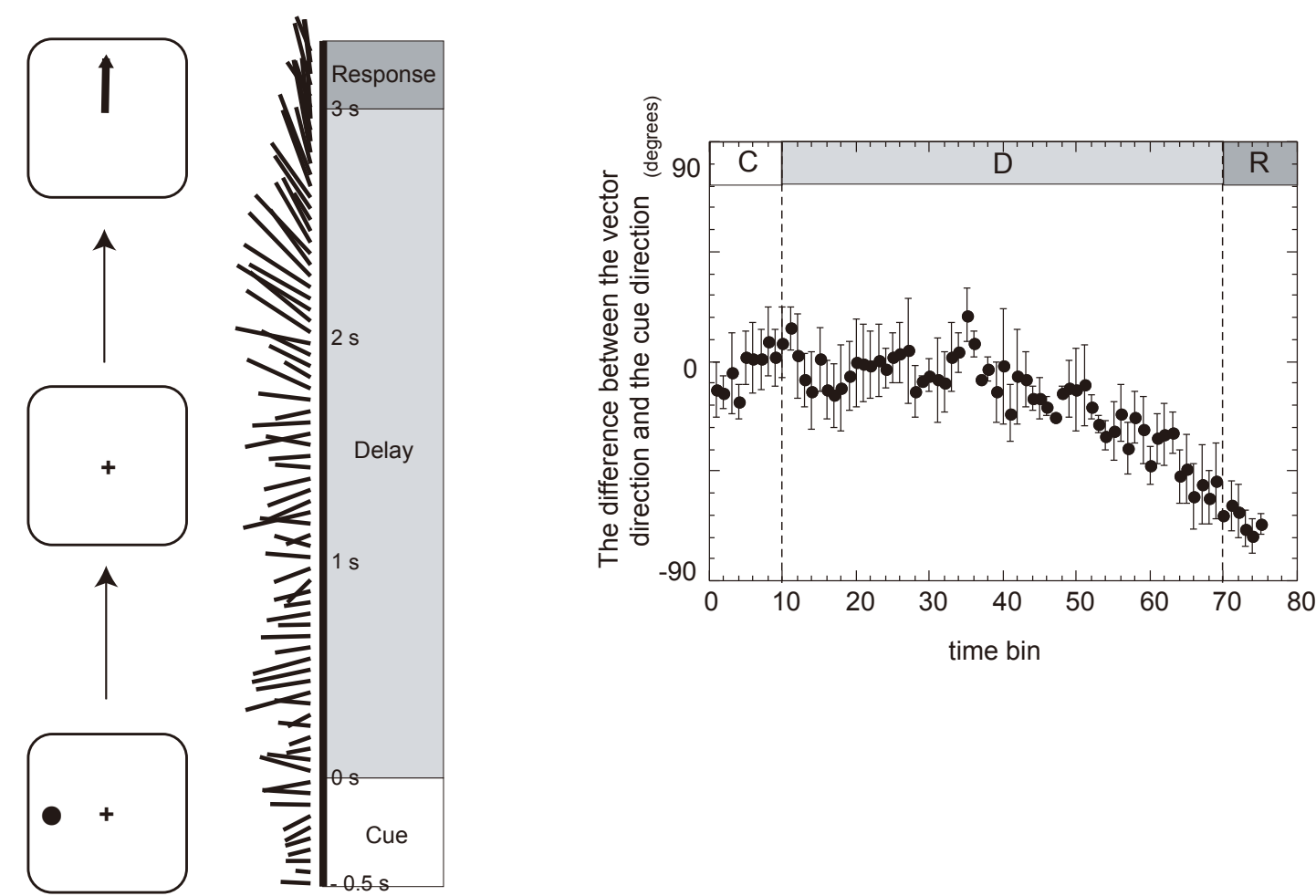


\section{A. Arrangements of fixation targets and visual cue locations}

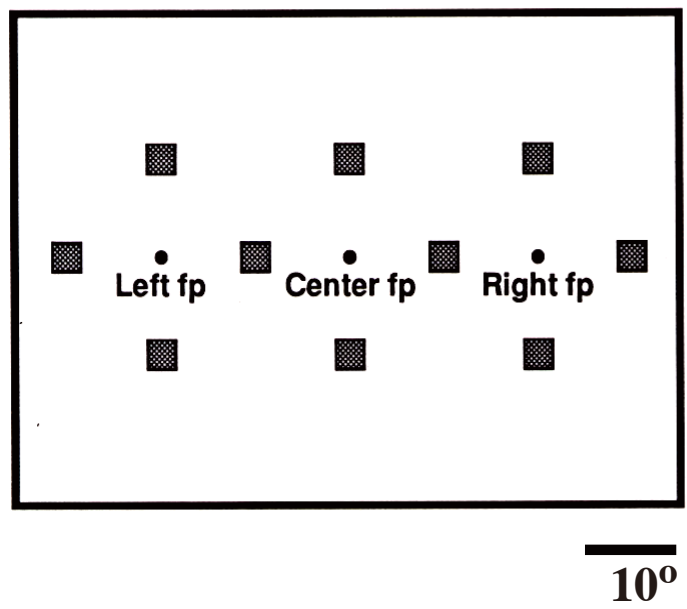

B. An example of prefrontal activity

Fixation conditions

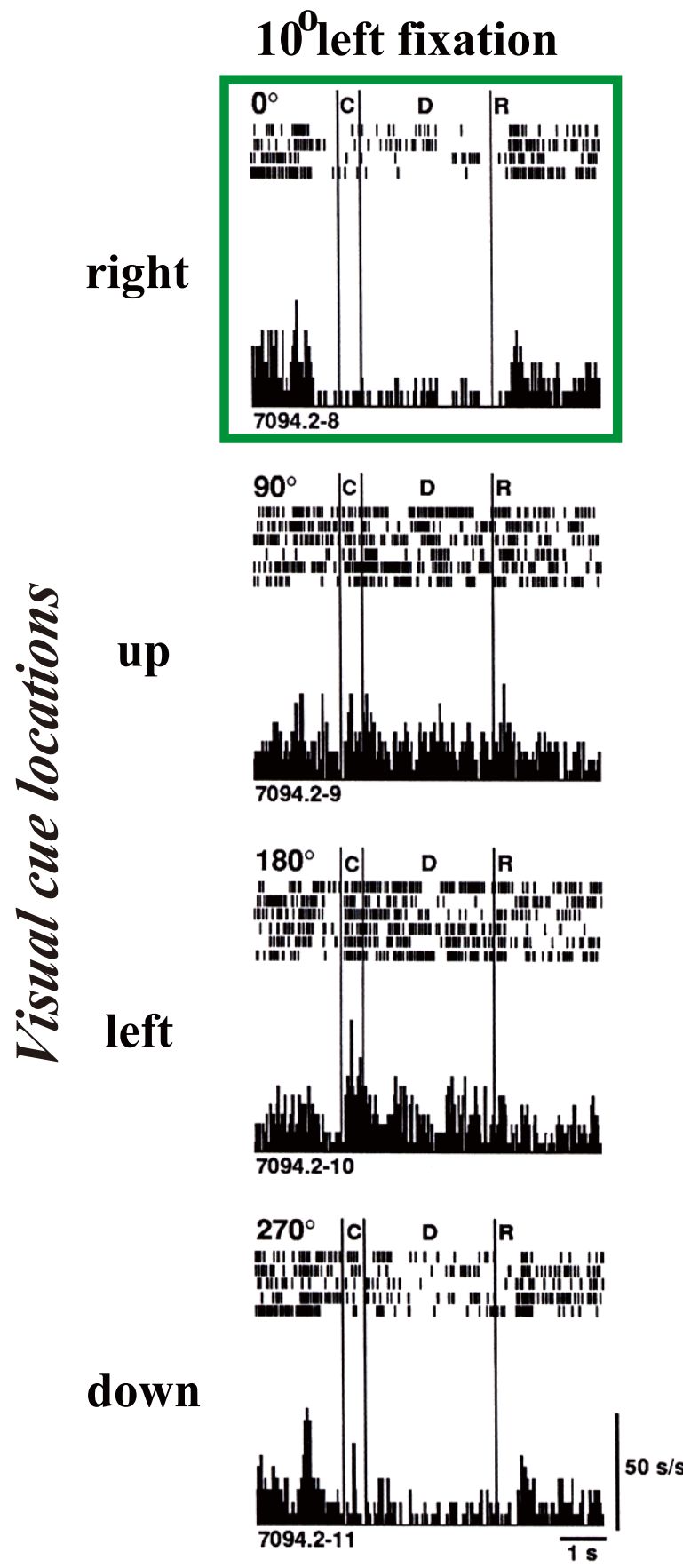

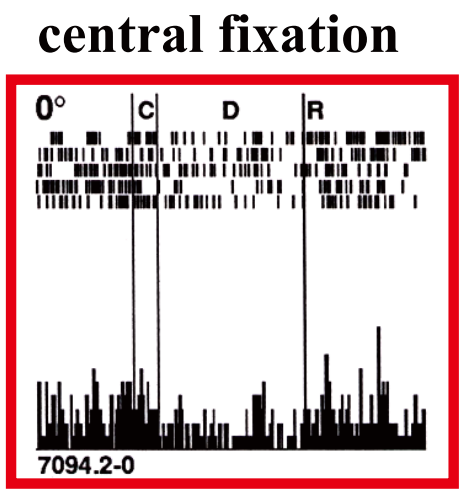
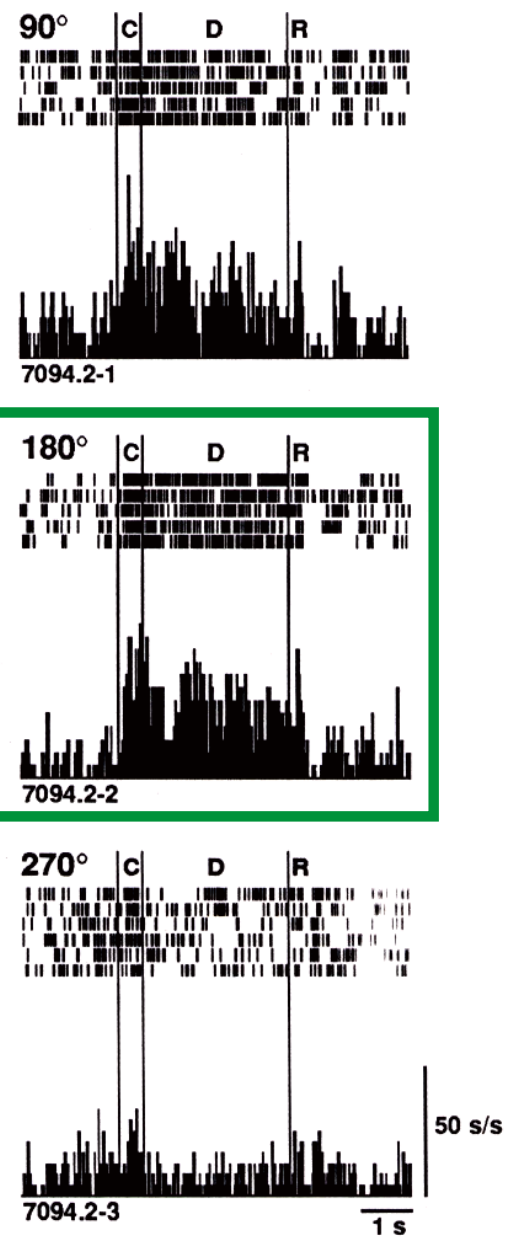

$10^{0}$ right fixation

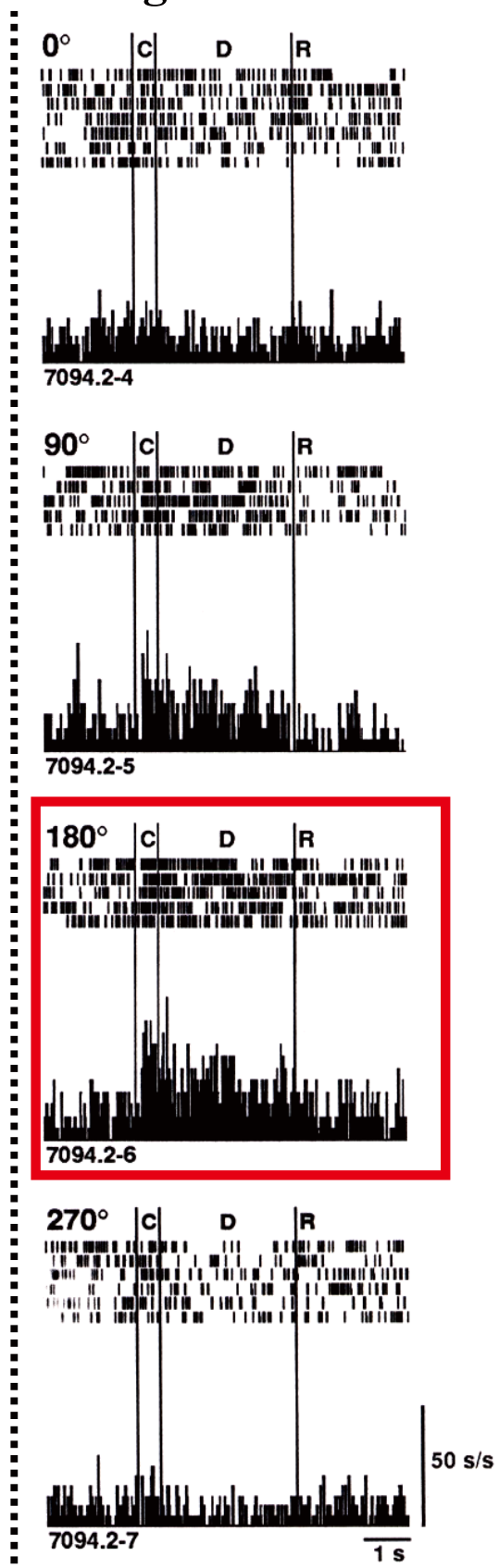




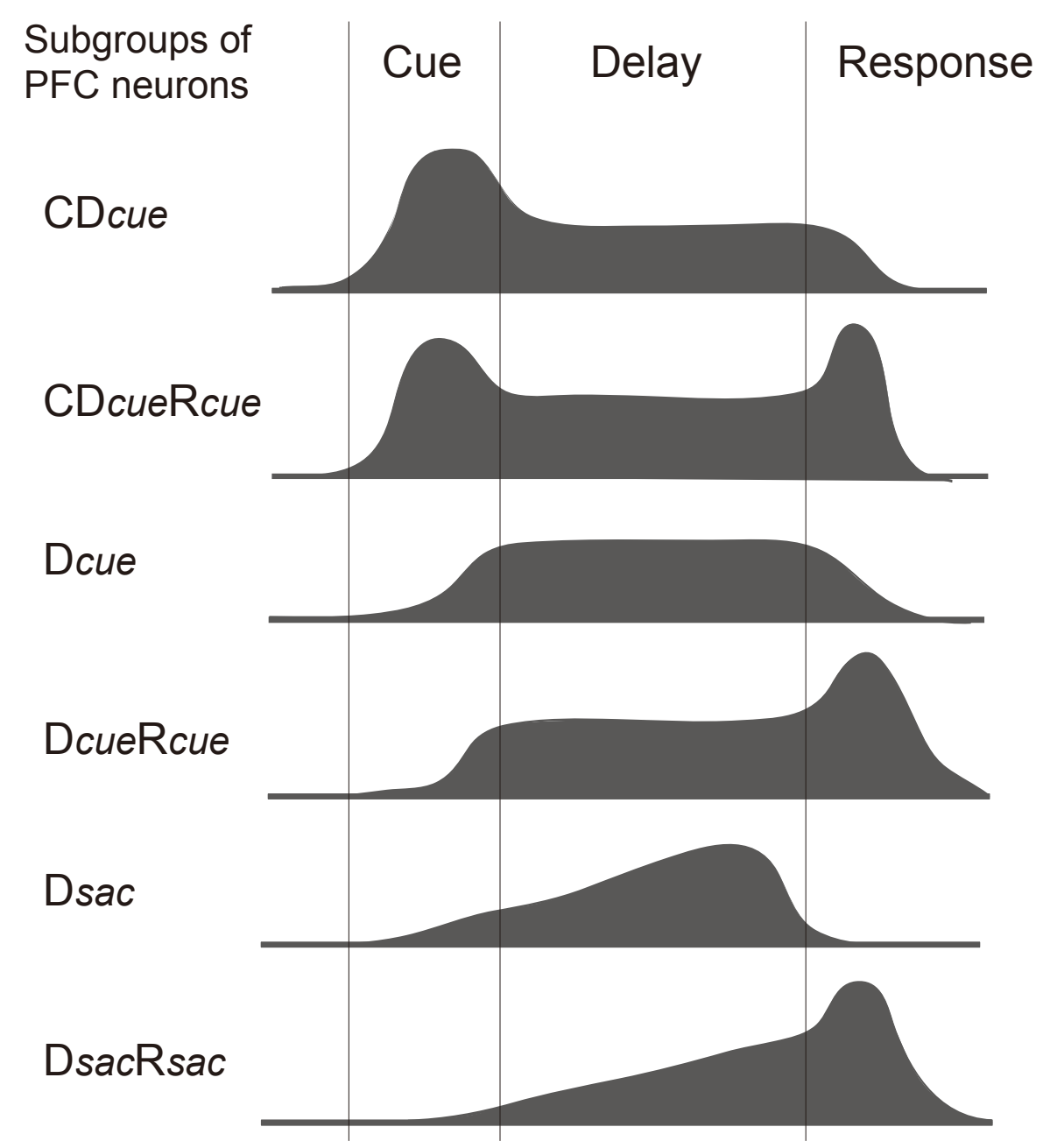




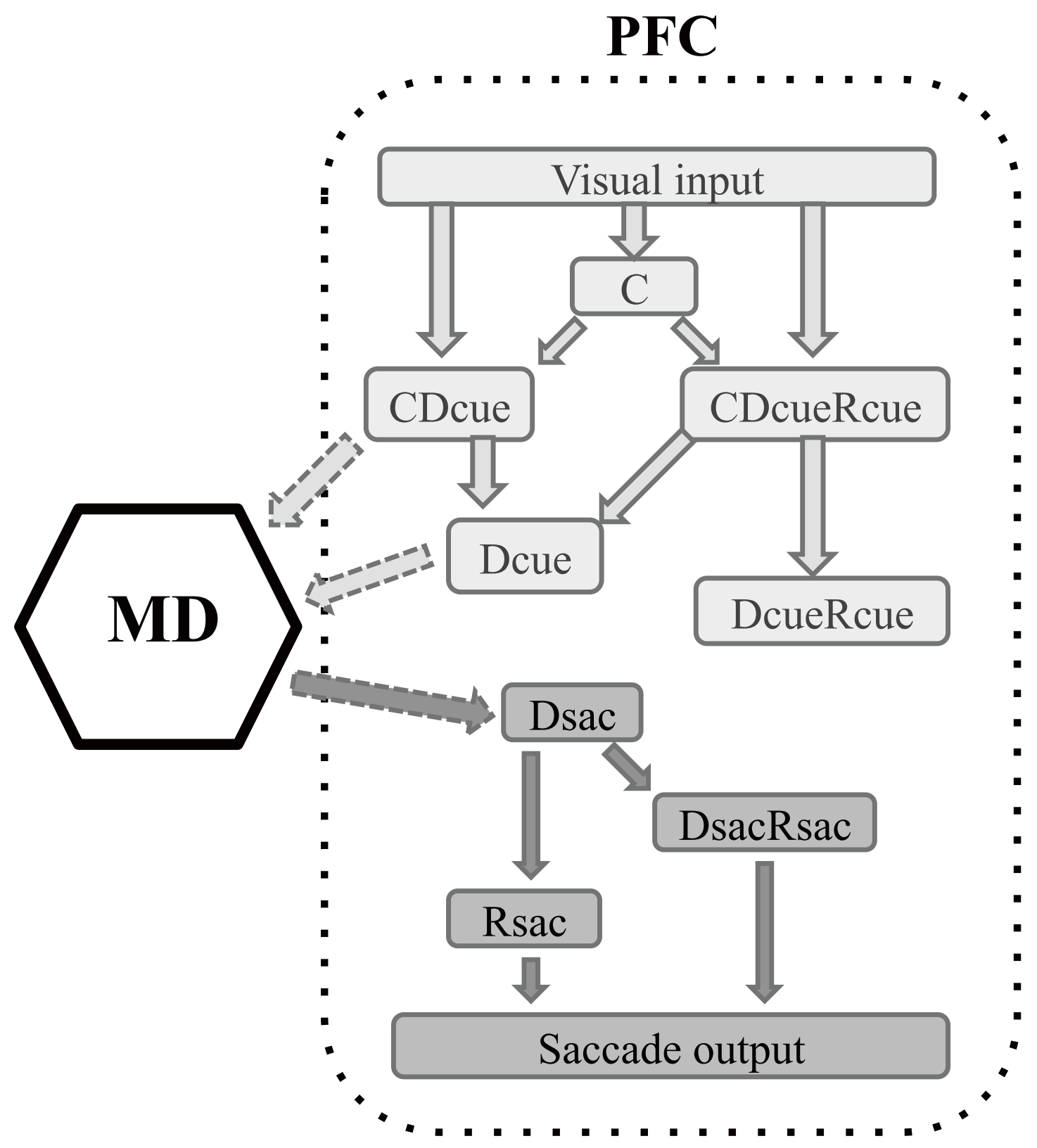

